\title{
Dynamic consistency, valuable information and subjective beliefs
}

\section{Spyros Galanis ${ }^{1}$}

Received: 16 October 2019 / Accepted: 11 February 2021 / Published online: 2 March 2021

(c) The Author(s) 2021

\begin{abstract}
Ambiguity sensitive preferences must fail either Consequentialism or Dynamic Consistency (DC), two properties that are compatible with subjective expected utility and Bayesian updating, while forming the basis of backward induction and dynamic programming. We examine the connection between these properties in a general environment of convex preferences over monetary acts and find that, far from being incompatible, they are connected in an economically meaningful way. In single-agent decision problems, positive value of information characterises one direction of DC. We propose a weakening of DC and show that one direction is equivalent to weakly valuable information, whereas the other characterises the Bayesian updating of the subjective beliefs which are revealed by trading behavior.
\end{abstract}

Keywords Updating · Ambiguity · Dynamic consistency · Bayesian · Consequentialism $\cdot$ Value of information

JEL Classification D81 · D83 · D91

\section{Introduction}

In dynamic-choice problems under uncertainty, the decision maker updates his preferences and his beliefs as new information arrives, taking optimal actions in each period. Two are the most widely used constraints on how these preferences are updated. The

This paper supersedes the paper "Dynamic Consistency and Subjective Beliefs". I am grateful to the editor and two referees, Alain Chateauneuf, Michèle Cohen, Martin Cripps, Adam Dominiak, Paolo Ghirardato, Jürgen Eichberger, Larry G. Epstein, Tassos Karantounias, Peter Klibanoff, Stelios Kotronis, Jean-Philippe Lefort, Jian Li, Sujoy Mukerji, Pietro Ortoleva, Luca Rigotti, Chris Shannon and audiences at CRETE 2014, ASSET 2014, RES 2015, RUD 2015, Cergy-Pontoise, Manchester, Warwick and Royal Holloway.

$凶$ Spyros Galanis

spyros.galanis@city.ac.uk

1 Department of Economics, City, University of London, London, UK 
first, Dynamic Consistency (DC), requires that an action plan is optimal when evaluated with the updated preferences of a later period if and only if it is optimal when evaluated with the preferences of an earlier period. DC ensures that an ex ante optimal action plan will remain optimal at every period and irrespective of how information is updated. The second, Consequentialism, requires that conditional preferences do not depend on past actions, foregone payoffs or unrealized events. These two properties form the basis of backward induction and dynamic programming.

The Subjective Expected Utility (SEU) model is consistent with both DC and Consequentialism, together with other attractive properties, such as positive value of information, recursive representation of preferences and Bayesian updating of beliefs. However, for other preferences this is not true in general. In particular, preferences which are ambiguity sensitive must either relax DC or Consequentialism (Siniscalchi 2009).

The purpose of this paper is to examine the connection between these properties in a general environment of convex preferences over monetary acts. We find that, far from being incompatible, they are connected in an economically meaningful way. We break DC into two parts. Consider two acts $f$ and $g$ which specify the same monetary payoffs if the complement of event $E$ occurs. The "if" direction of DC specifies that if the agent weakly prefers $f$ over $g$ ex ante, then he also prefers it when he learns that $E$ occurred. The "only if" direction requires the converse.

We first show that the "only if" direction of DC is inconsistent with Ellsberg (1961) but equivalent to positive value of information, meaning that a single agent always prefers to receive more information to less. Intuitively, an agent who understands that his preferences will change if he learns an event might not want to receive this information, as this could lead to choices that he considers suboptimal in the ex ante stage. Although several authors have previously discussed this connection, to our knowledge this is the first paper that provides a formal characterization.

Al-Najjar and Weinstein (2009) have criticised the ambiguity aversion literature on the basis that aversion to information is not normatively appealing. However, we show that ambiguity averse preferences are consistent with a weaker version of valuable information. In particular, we say that information is weakly valuable if (ex ante) the agent always prefers mixing more information with less information, rather than receiving less information with certainty. Such an agent recognises that more information has at least some value, even if he does not think that more information with certainty is optimal. We then characterize weakly valuable information with respect to a weakening of the "only if" direction of DC. These results on the value of information do not require the full strength of Consequentialism (although they are still true when Consequentialism is assumed). In particular, we use a weaker axiom, Status Quo Bias. This property was first proposed in axiomatic work by Masatlioglu and Ok (2005), however it has been studied experimentally at least since Samuelson and Zeckhauser (1988), who provided evidence in a study concerning portfolio choices.

Second, we show that a weakening of the "if" direction of DC characterizes the Bayesian updating of the beliefs revealed by potential trading behavior (and are not necessarily part of the utility representation of preferences). They are called "subjective beliefs" by Rigotti et al. (2008) (RSS), who identify them for a wide variety of models with convex preferences over monetary acts, hence making our approach very general. 
Subjective beliefs have economic content in static environments, as RSS show that they characterize efficient and full insurance allocations. Moreover, each subjective belief can be interpreted as state prices of Arrow-Debreu securities, for which the agent is not willing to trade the act $f$ with which he is endowed. ${ }^{1}$ If these prices prevail in the market, we say that $f$ is revealed preferred to all the acts that are affordable for the agent. Our proposed weakening of the "if" direction of DC specifies that if $f$ is ex ante revealed preferred (but not necessarily weakly preferred) to another act $g$ then $f$ is ex post weakly preferred to $g$. The weakening of the "only if" direction of DC specifies that if $f$ is ex post weakly preferred to $g$, then it cannot be that $g$ is ex ante revealed preferred (but could be strictly preferred) to $f .^{2}$

Using the results of RSS, who characterise the subjective beliefs for several ambiguity averse preference models, we discuss some updating rules. We note that our domain of preferences, just like in RSS, is acts from states to monetary outcomes, a special case of Savage (1954). This is the natural domain in order to interpret subjective beliefs as prices of Arrow-Debreu securities and motivate the weakening of DC using the notion of revealed preference. However, many decision theoretic models use the more general domain of acts from states to lotteries over outcomes. We discuss in Sect. 6 how weak DC extends to this domain.

In a companion paper (Galanis 2021), we show that the economic content of subjective beliefs extends to dynamic and multi-agent environments. First, their Bayesian updating is the minimum requirement which ensures that there is no speculative trade, generalising the result of Milgrom and Stokey (1982). Second, if information is (weakly) valuable for each agent, then public information is (weakly) not valuable, generalising the result of Hirshleifer (1971) and Schlee (2001) in competitive risk-sharing environments without aggregate uncertainty.

\subsection{Our approach}

We illustrate our approach by analyzing a dynamic Ellsberg's three-color problem, taken from Epstein and Schneider (2003) and Hanany and Klibanoff (2007). We show that Ellsberg preferences, Consequentialism and an axiom we call Conditional Preference imply that DC and value of information are violated. We then explain our weakening of DC.

An urn contains 120 balls, 40 of which are known to be black (B), whereas the remaining 80 are somehow divided between red $(\mathrm{R})$ and yellow (Y). The state space is $S=\{B, R, Y\}$. A bet is an act from $S$ to $\mathbb{R}$, specifying a payoff at each state.

Consider acts $f_{1}=(1,0,0), f_{2}=(0,1,0), f_{3}=(0,1,1)$ and $f_{4}=(1,0,1)$, where, for example, $f_{1}$ specifies a payoff of 1 if the state is $B$ and 0 otherwise. The agent has ex ante preferences $\succsim$ which conform to Ellsberg, hence $f_{1} \succ f_{2}$ and $f_{3} \succ f_{4}$.

\footnotetext{
${ }^{1}$ An act can be identified by a convex combination of Arrow-Debreu securities, thus providing a mapping from states to consumption of a single good.

${ }^{2}$ As with DC, the axiom applies only for acts $f$ and $g$ which are identical outside of the event that is revealed ex post. Formal details are presented in Sect. 2.
} 
We assume that the agent is endowed with preference relation $\succsim_{\{B, R\}}$ conditional on learning that event $\{B, R\}$ has occurred and $\succsim_{\{Y\}}$ conditional on learning that event $\{Y\}$ has occurred. This specification implicitly assumes Consequentialism, because the conditional preference only depends on the updated event and not on other parameters, such as the act that the agent chose in the previous period or the decision problem he faced. In the rest of the paper, we relax this assumption, so that conditional preferences may depend on the event and the act that was chosen in the previous period.

Note that, conditional on $\{Y\}, f_{1}$ is identical to $f_{2}$ and $f_{3}$ is identical to $f_{4}$. The axiom Conditional Preference requires that the agent is indifferent between such acts if he learns that $\{Y\}$ has occurred, hence $f_{1} \sim_{\{Y\}} f_{2}$ and $f_{3} \sim_{\{Y\}} f_{4}{ }^{3}$ Similarly, because conditional on $\{B, R\} f_{1}$ is identical with $f_{4}$ and $f_{2}$ is identical with $f_{3}$, Conditional Preference requires that $f_{1} \sim_{\{B, R\}} f_{4}$ and $f_{2} \sim_{\{B, R\}} f_{3}$.

We first show that these Ellsberg preferences imply that DC is violated and information is not valuable. We represent information by a partition $\Pi$ of $S$. Let $\mathcal{A}=\left\{f_{3}, f_{4}\right\}$ be the set of feasible acts. Without loss of generality, suppose that $f_{4} \succsim_{\{B, R\}} f_{3} .{ }^{4}$ First, suppose that the agent has no information, so his partition is $\Pi_{1}=\{S\}$. In other words, he never learns whether $\{Y\}$ has occurred or not, so $\succsim_{\{Y\}}$ and $\succsim_{\{B, R\}}$ are irrelevant. Then, he chooses $f_{3}$, because $f_{3} \succ f_{4}$.

Consider now the more informative partition $\Pi_{2}=\{\{B, R\}, Y\}$, meaning that he is informed whether $Y$ has occurred or not, before making his choice. If he learns that $\{B, R\}$ has occurred, he chooses $f_{4}$ because $f_{4} \succsim_{\{B, R\}} f_{3}$, whereas if he learns $\{Y\}$, he again chooses $f_{4}$ because $f_{3} \sim_{\{Y\}} f_{4}$.

The agent understands that if his partition is $\Pi_{1}$ he will get $f_{3}$ in all states, whereas if his partition is $\Pi_{2}$ he will get $f_{4}$. He strictly prefers the former ex ante because $f_{3} \succ f_{4}$. Because $\Pi_{2}$ is finer than $\Pi_{1}$, we say that information is not valuable. The "only if" direction of DC (Axiom 12) specifies that if the agent weakly prefers $f_{4}$ over $f_{3}$ given that he has learned $\{B, R\}$ and the acts are identical outside of $\{B, R\}$, then he also weakly prefers $f_{4}$ over $f_{3}$ before learning whether $\{B, R\}$ has occurred or not. This is violated here as we have both $f_{4} \succsim_{\{B, R\}} f_{3}$ and $f_{3} \succ f_{4}$. Violation of both Axiom 12 and valuable information is not particular to this example, as Proposition 1 shows that they are equivalent.

The above results show that we cannot weaken the "only if" part of DC without losing positive value of information. Suppose now that $a f_{4}+(1-a) f_{3} \succsim f_{3}$ for some $a \in(0,1]$. Then, the agent recognises that more information has some value, because he would prefer to mix more with less information, instead of getting less information for sure. ${ }^{5}$ When this happens, we say that information is weakly valuable.

\footnotetext{
${ }^{3}$ Several authors call this property Consequentialism, implicitly assuming that the conditional preference only depends on the realised event. Following Epstein and Schneider (2003), we call this property Conditional Preference and formally define it in Sect. 2 as Axiom 5. We assume it throughout the paper.

${ }^{4}$ For the case where $f_{3} \succsim_{\{B, R\}} f_{4}$, we can obtain negative value of information using a similar example, with feasible acts $\mathcal{A}=\left\{f_{1}, f_{2}\right\}$, as Consequentialism implies that $f_{2} \succsim_{\{B, R\}} f_{1}$.

5 The interpretation of mixing depends on the range of acts. If the range is monetary outcomes (or more generally a convex set, a special case of Savage (1954)), then $a f_{4}+(1-a) f_{3}$ provides, at state s, the monetary outcome $a f_{4}(s)+(1-a) f_{3}(s)$. If acts are contingent lotteries, a special case of Anscombe and Aumann (1963), then $a f_{4}(s)+(1-a) f_{3}(s)$ is a lottery that gives $f_{4}(s)$ with probability $a$ and $f_{3}(s)$ with probability $1-a$. In the paper the range is monetary outcomes, but we discuss the extension to state contingent lotteries in Sect. 6.
} 




Fig. 1 Revealed preference

Proposition 1 characterizes this form of valuable information in terms of Axiom 14, which is weaker than Axiom 12, the "only if" part of DC.

We weaken DC by introducing the revealed preference relation, denoted $\succsim^{*}$. We say that $f$ is revealed preferred to $g$ if $f$ is weakly preferred to all mixtures $a f+(1-a) g$, for $a \in[0,1]$. This is neither a complete nor a transitive preference relation. In Fig. 1, there are two states, $s_{1}$ and $s_{2}$ and a point denotes an act. The depicted indifference curve has a kink at $f$ and several supporting hyperplanes, given by the shaded area. Although $f$ is strictly preferred to $g, h$ and $k$, it is revealed preferred only to $g$ and $h$. Even though $f$ is not revealed preferred to $k$, convexity of preferences implies that $f$ is strictly preferred to some convex combination of $f$ and $k$. This convexity property is used extensively.

The normals of the hyperplanes that pass from $f$ (normalized to be probability distributions) are called subjective beliefs at $f$ by Rigotti et al. (2008) and denoted by $\pi(f)$. For convex preferences, each $p \in \pi(f)$ has the following property: if the expectation of $f$ given $p$ (denoted $\mathbb{E}_{p} f$ ) is greater or equal to the expectation of another act $g$ given $p$, then $f$ is weakly preferred to $g$. We can interpret $p$ as a price vector, such that $p(s)$ is the price of the Arrow-Debreu security that pays 1 if state $s$ occurs and 0 otherwise. Then, the property says that if the agent's endowment is $f$, he would have zero net demand at each price vector $p \in \pi(f)$. Hence, we say that $f$ is revealed preferred to $g$ because the agent could afford $g$ given prices $p$ but chose his endowment, $f$.

Suppose that acts $f$ and $g$ are identical in terms of what they prescribe if event $E$ does not occur. Then, the "if" part of DC is weakened by requiring that if $f$ is revealed preferred (but not necessarily weakly preferred) to $g$ ex ante, then $f$ is weakly preferred to $g$ conditional on $E$. The "only if" part of DC is weakened by requiring that if $f$ is weakly preferred to $g$ conditional on $E$, then $g$ cannot be revealed preferred (but could be strictly preferred) to $f$ ex ante. 
In this section, we have assumed Consequentialism. In the rest of the paper we relax this, so that conditional preferences can depend both on the event and on the act that was chosen in the ex ante stage.

The paper proceeds as follows. Section 2 presents the model, whereas Sect. 3 formalises the notions of (weak) valuable information and characterizes them with respect to the "only if" part of (weak) DC. In Sect. 4, we characterize the "if" part of weak DC with respect to Bayesian updating of subjective beliefs. We examine various updating rules in Sect. 5. In Sect. 6, we discuss the related literature and provide a detailed comparison with Ghirardato et al. (2004) and Hanany and Klibanoff (2007, 2009), which are more closely related with our approach. All proofs are contained in the Appendix.

\section{Model}

\subsection{Preliminaries}

Fix a finite set of payoff relevant states $S$, with typical element $s$. The set of consequences is $\mathbb{R}_{+}$, interpreted as monetary payoffs. Let $\mathcal{F}=\mathbb{R}_{+}^{S}$ be the set of acts, with the natural topology. An act $f \in \mathcal{F}$ maps each state $s$ to a monetary payoff. Given $x \in \mathbb{R}_{+}$, let $x \in \mathcal{F}$ be the constant act with payoff $x$ at each state $s$. Let $X$ be the set of constant acts. An act $f$ is strictly positive if $f(s)>0$ for all $s \in S$. Let $\mathcal{F}_{+}$be the set of strictly positive acts.

For any two acts $f, g \in \mathcal{F}$ and event $E \subseteq S$, we denote by $f E g$ the act $h$ such that $h(s)=f(s)$ if $s \in E$ and $h(s)=g(s)$ if $s \notin E$. Define $f \geq_{E} g$ if $f(s) \geq g(s)$ for all $s \in E$, with strict inequality for some $s \in E$. Equality $f={ }_{E} g$ and strict inequality are similarly defined. Let $E^{c}$ be the complement of $E$ with respect to $S$.

Given events $E, F \subseteq S$ and probability measure $p \in \Delta E$, where $F \subseteq E$ and $p(F)>0$, denote by $p_{F} \in \Delta F$ the measure obtained through Bayesian conditioning of $p$ on $F$. Formally, for any event $G \subseteq S, p_{F}(G)=\frac{p(G \cap F)}{p(F)}$. We write $\mathbb{E}_{p} f:=$ $\sum_{s \in E} p(s) f(s)$ for the expectation of $f$ given $p$.

Let $\mathcal{E}$ be a collection of nonempty events $E \subseteq S$ which contains $S$. The decision maker is endowed with a collection of conditional preference relations, $\left\{\succsim_{E, h}\right.$

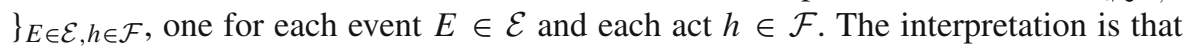
in a previous period the agent had chosen act $h$ and in the current period he learns that event $E$ has occurred. His updated preference relation is then $\succsim_{E, h}$. The ex ante preference relation $\succsim_{S, h}$ does not depend on the act $h$ and is denoted by $\succsim$.

A partition $\Pi$ of $S$ is a collection of mutually disjoint events, whose union is $S$. It is finer than another partition $\Pi^{\prime}$ if, for each $E^{\prime} \in \Pi^{\prime}$, there exists $E \in \Pi$ with $E \subseteq E^{\prime}$. We then say that $\Pi^{\prime}$ is coarser than $\Pi$. 


\subsection{Revealed preference}

Given preference relation $\succsim_{E, h}$, we say that act $f$ is revealed preferred to act $g$, written $f \succsim_{E, h}^{*} g$, if $f \succsim_{E, h} a g+(1-a) f$ for all $a \in[0,1]$, so that $f$ is weakly preferred to all convex combinations of $f$ and $g$. Preference relation $\succsim_{E, h}^{*}$ is neither transitive nor complete.

The interpretation of $f \succsim_{E, h}^{*} g$ is that $f$ is weakly preferred to $g$ under $\succsim_{E, h}$ and $g$ is inside a "budget set", which is constructed given $f$ as the agent's endowment and some prices for the Arrow-Debreu securities, one for each state. If these prices were to prevail and the agent chose $f$, it would be revealed that the agent prefers $f$ over $g$. Consider Fig. 1. The indifference curve has a kink on act $f$ and the two straight lines which define the shaded area are some of its supporting hyperplanes. Each such line defines a budget set, where $f$ is affordable. Any act that is within this budget set, like $g$, is affordable but $f$ is weakly preferred to $g$, hence we say that $f$ is revealed preferred to $g$. On the contrary, although $f$ is strictly preferred to $k$, it is not revealed preferred to it, because it is outside any of these budget sets.

\subsection{Convex preferences}

We consider the following axioms on preferences $\left\{\succsim_{E, h}\right\}_{E \in \mathcal{E}, h \in \mathcal{F}}$, for all events $E \in \mathcal{E}$ and acts $h \in \mathcal{F}$.

Axiom 1 (Preference). $\succsim_{E, h}$ is complete and transitive.

Axiom 2 (Continuity). For all $f \in \mathcal{F}$, the sets $\left\{g \in \mathcal{F}: g \succsim_{E, h} f\right\}$ and $\{g \in \mathcal{F}$ : $\left.f \succsim_{E, h} g\right\}$ are closed.

Axiom 3 (Monotonicity). For all $f, g \in \mathcal{F}$, if $f>_{E} g$ then $f \succ_{E, h} g$.

Axiom 4 (Convexity). For all $f \in \mathcal{F}$, the set $\left\{g \in \mathcal{F} \mid g \succsim_{E, h} f\right\}$ is convex.

These four axioms are standard and imply that each $\succsim_{E, h}$ is represented by a continuous, increasing and quasiconcave function $U_{E, h}: \mathcal{F} \rightarrow \mathbb{R}$. The next axiom, which we require throughout the paper, specifies that if the agent knows that event $E$ has occurred, his preferences depend only on what acts specify inside $E$.

Axiom 5 (Conditional Preference) For all $f, g \in \mathcal{F}$, if $f={ }_{E} g$ then $f \sim_{E, h} g$. 5 .

We say that preferences $\left\{\succsim_{E}\right\}_{E \in \mathcal{E}, h \in \mathcal{F}}$ are convex if they satisfy Axioms 1 through

Axiom 6 (Strict Convexity). For all $f \neq_{E} g$ and $\alpha \in(0,1)$, if $f \succsim_{E, h} g$, then $\alpha f+(1-\alpha) g \succ_{E, h} g$.

The following axiom, No Flat Kinks, is weaker than Strict Convexity and specifies that if $g$ is strictly preferred to $f$, then it is not the case that $g$ is indifferent to all convex combinations of $f$ and $g$ for a closed interval of weights. We use this axiom in order to show the equivalence of weakly valuable information and one direction of weak DC. It allows for straight indifference curves but not those that have a "flat" kink. 
Axiom 7 (No Flat Kinks) If $g \succ_{E, h} f$ then there does not exist $\bar{a} \in(0,1]$ such that $a f+(1-a) g \sim_{E, h} g$ for all $a \in[0, \bar{a}]$.

An event $F \subseteq E$ is non $\succsim_{E, h}$-null if $g(s)>g^{\prime}(s)$ for all $s \in F$ implies $g F f \succ_{E, h}$ $g^{\prime} F f$, for all acts $g, g^{\prime}, f$. Hanany and Klibanoff $(2007,2009)$ restrict attention to non-null events. ${ }^{6}$ However, we impose a weaker definition of non-nullity, which is motivated by the property that all subjective beliefs assign positive probability to not

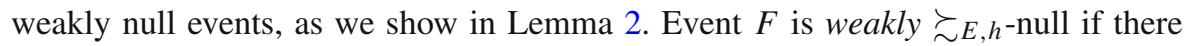
exists act $g$ such that, for all acts $g^{\prime}, f, g F f \succsim_{E, h} g^{\prime} F f$. Because for every act $f$ there exists another act $g$ such that $g(s)>f(s)$ for all $s \in F$, we have that if $F$ is non-null then it is also not weakly null. The following axiom requires that all events in $\mathcal{E}$ are not weakly null.

Axiom 8 (Weak Full Support). For all events $E, F \in \mathcal{E}$, where $F \subseteq E, F$ is not weakly $\succsim_{E, h}$-null.

\subsection{Consequentialism}

Consequentialism requires that the agent's preferences depend only on the received information and not on the act that was chosen in the previous period. ${ }^{7}$

Axiom 9 (Consequentialism) For all $f, g \in \mathcal{F}$ and events $E \in \mathcal{E}, \succsim_{E, f}=\succsim_{E, g}$.

A weakening of Axiom 9 has been proposed in axiomatic work by Masatlioglu and Ok (2005), Sagi (2006) and Ortoleva (2010), where preference relation $\succsim_{E, h}$ depends on a "status quo" act (or frame) $h$. It specifies that if the agent ever prefers $f$ over $g$ (given some status quo $h$ ), then he would also prefer it if the status quo was $f$. In other words, the status quo exerts attraction towards itself.

Axiom 10 (Status Quo Bias) For all $f, g, h \in \mathcal{F}$ and events $E \in \mathcal{E}$, if $f \succsim_{E, h} g$ then $f \succsim E, f g$.

As pointed by Masatlioglu and Ok (2005), Status Quo Bias is documented not only by experimental studies but also by empirical work in actual markets. For instance, Madrian and Shea (2001) examined how the default choice influenced participation in 401(k) saving plans, whereas Samuelson and Zeckhauser (1988) identified Status Quo Bias experimentally, in a study concerning portfolio choices.

\subsection{Dynamic consistency}

DC provides restrictions on how two acts, which are identical outside of the conditioning event $E$, should be compared before and after $E$ is known to have occurred. We break DC into two Axioms and adopt the names proposed by Ghirardato (2002).

\footnotetext{
${ }^{6}$ In their setting, $F$ is non $\succsim_{E, h}$-null if $g(s) \succ_{E, h} g^{\prime}(s)$ for all $s \in F$ implies $g F f \succ_{E, h} g^{\prime} F f$, for all acts $g, g^{\prime}, f$.

${ }^{7}$ Some papers refer to Consequentialism as the conjunction of Axioms 5 and 9.
} 
Axiom 11 (Consistency of Implementation) For all acts $f, g \in \mathcal{F}$ and events $E \in \mathcal{E}$, if $f \succsim g$ and $f=E^{c} g$ then $f \succsim_{E, f} g$.

Suppose that $f$ and $g$ specify the same payoff at each state not belonging to event $E$ and that $f$ is weakly preferred to $g$ ex ante. Consistency of Implementation says that if the agent has chosen $f$ ex ante and he is informed that event $E$ has occurred (so that his preferences are $\succsim_{E, f}$ ), then in the interim stage $f$ is still weakly preferred to $g$. The next axiom is the converse. If $f$ is weakly preferred to $g$ in the interim stage, given event $E$ and preferences $\succsim_{E, f}$, then $f$ is weakly preferred to $g$ in the ex ante stage.

Axiom 12 (Information is Valuable) For all acts $f, g \in \mathcal{F}$ and events $E \in \mathcal{E}$, if $f \succsim_{E, f} g$ and $f=E_{E^{c}} g$ then $f \succsim g$.

\subsection{Weak dynamic consistency}

Using our notion of revealed preference, we provide a weakening of DC. Axiom 11 (Consistency of Implementation) is weakened by requiring that if ex ante $f$ is revealed preferred (but not necessarily weakly preferred) to $g$, then $f$ is weakly preferred to $g$, conditional on $E$. We also require that $f$ is a strictly positive act.

Axiom 13 (Weak Consistency of Implementation) For all acts $f \in \mathcal{F}_{+}, g \in \mathcal{F}$ and events $E \in \mathcal{E}$, if $f \succsim^{*} g$ and $f=E^{c} g$ then $f \succsim_{E, f} g$.

Suppose that there are three states $\left\{s_{1}, s_{2}, s_{3}\right\}$, the graph in Fig. 1 depicts acts that are identical given $s_{3}$ but the indifference curve represents the ex ante preference relation $\succsim$, where $S=\left\{s_{1}, s_{2}, s_{3}\right\} .{ }^{8}$ Act $f$ is strictly preferred to $g, h$ and $k$ according to $\succsim$ but it is revealed preferred only to $g$ and $h$. Hence, Axiom 13 requires that, given $E=\left\{s_{1}, s_{2}\right\}, f$ is weakly preferred to $g$ and $h$ but not $k$. Proposition 2 shows that Axiom 13 characterizes Bayesian updating of subjective beliefs.

The other direction of DC, Axiom 12 (Information is Valuable), is weakened in a similar manner. Axiom 14 requires that if $f$ is weakly preferred to $g$ conditional on $E$ and $f$ but ex ante strictly preferred to $f$, then $g$ is not revealed preferred to $f$.

Axiom 14 (Weak Information is Valuable) For all acts $f \in \mathcal{F}, g \in \mathcal{F}_{+}$and events $E \in \mathcal{E}$, if $f \succsim_{E, f} g, f=E_{E^{c}} g$ and $g \succ f$ then $g \chi^{*} f$.

That is, unlike Axiom 12 (Information is Valuable), Axiom 14 allows for a preference reversal, from $f \succsim_{E, f} g$ to $g \succ f$, but not so severe that we also have $g \succsim^{*} f$. In the previous example, if $k \succsim_{E, k} f$ and $k=E_{E^{c}} f$, then Axiom 14 requires that $f \beth^{*} k$, so that $k$ lies strictly above the shaded area. This means that Axiom 14 allows for $f \succ k$, as is shown in Fig. 1, unlike Axiom 12 (Information is Valuable) which requires that $k \succsim f$ and therefore is stronger for convex preferences. Proposition 1 shows that Axiom 14 is equivalent to weakly valuable information.

What is the connection between the two parts of DC and weak DC? Under Strict Convexity and Status Quo Bias, (Weak) Information is Valuable implies (Weak) Consistency of Implementation. If we strengthen Status Quo Bias to Consequentialism, then the converse is also true.

\footnotetext{
8 Axiom 13 has content if there are at least three states.
} 


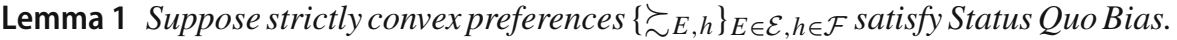
Then, (Weak) Value of Information implies (Weak) Consistency of Implementation. Under Consequentialism, the converse is also true.

\section{Valuable information}

Several papers show with examples that failure of DC implies that information is not always valuable, under various settings (e.g. Wakker (1988), Epstein and Le Breton (1993)). In this section, we provide a formal treatment of this result and show that the converse is also true. In particular, we define information to be valuable if an agent always ex ante prefers having a finer than a coarser partition, given that he will choose an action in the interim stage, when a partition cell is revealed to him. We show that valuable information characterises the second part of DC (Axiom 12). ${ }^{9}$

However, even if the agent does not think that more information is always valuable, he may still recognise that it has some value. If the agent prefers mixing, for some $a \in(0,1]$, between receiving the finer and the coarser partition, over receiving the coarser partition with certainty, we say that information is weakly valuable and show that it characterizes Axiom 14 (Weak Information is Valuable), which is the second part of weak DC.

In order to define (weakly) valuable information, we adapt the framework of Geanakoplos (1989), which assumed expected utility, to the present setting. There are two periods, 0 and 1. For simplicity, initially assume Axiom 9 (Consequentialism). An agent faces some uncertainty in period 0 , represented by ex ante preferences $\succsim$ and a finite state space $S$. His decision problem consists of a feasible set of acts, $\mathcal{A}$, and a partition $\Pi=\left\{E_{1}, \ldots, E_{n}\right\}$ of $S$. The agent expects that, in period 1, he will be informed that a particular cell $E \in \Pi$ of his partition has occurred and will choose an "interim" act $f_{E} \in \mathcal{A}$, which is optimal according to his conditional preferences $\succsim_{E}$. By choosing an optimal interim act $f_{E}$ for each partition cell $E \in \Pi$, he can generate an "ex-ante" optimal act $h$ such that $h={ }_{E} f_{E}$, for each $E \in \Pi$. In other words, the ex ante optimal act $h$ agrees with the interim acts $f_{E_{1}}, \ldots f_{E_{n}}$, conditional on each element of $\Pi$, and $f_{E} \succsim_{E} g$ for all $g \in \mathcal{A}$.

Since we want to characterise valuable information also in the case where Consequentialism is not assumed, we adjust slightly the definition of ex ante optimality. In particular, we say that the ex ante optimal act $h$ agrees with the interim acts $f_{E_{1}}, \ldots f_{E_{n}}$, conditional on each element of $\Pi$, and $f_{E} \succsim_{E, h} g$ for all $g \in \mathcal{A}$.

The ex ante optimal act may not be unique, because there may be many interim optimal acts $f_{E}, f_{E}^{\prime} \in \mathcal{A}$ given $E$. We therefore require, in order to say that partition $\Pi$ is "more valuable" than partition $\Pi^{\prime}$ given $\mathcal{A}$, that for every ex ante optimal act $h^{\Pi^{\prime}}$ for $\Pi^{\prime}$ there is an ex ante optimal act $h^{\Pi}$ for $\Pi$ such that $h^{\Pi} \succsim h^{\Pi^{\prime}}$. It is weakly more valuable if $a h^{\Pi}+(1-a) h^{\Pi^{\prime}} \succsim h^{\Pi^{\prime}}$, for some $a \in(0,1]$. We then say that information is (weakly) valuable if, for all $\mathcal{A}$, whenever $\Pi$ is finer than $\Pi^{\prime}$ it is also (weakly) more valuable.

\footnotetext{
9 We are not aware of such a characterization previously shown in the literature.
} 
We now provide the formal treatment. Fix a collection of conditional preference

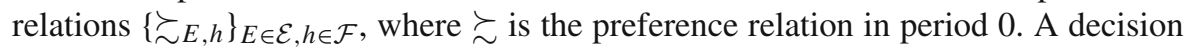
problem $\mathcal{D}=\{\Pi, \mathcal{A}\}$ consists of a partition $\Pi \subseteq \mathcal{E}$ of $S$ representing the information in period 1 and a set of available acts $\mathcal{A} \subseteq \mathcal{F}$.

An act $f \in \mathcal{F}$ is feasible ex ante for decision problem $\mathcal{D}$ if for each $E \in \Pi$ there exists $g \in \mathcal{A}$ such that $f={ }_{E} g$, so that they provide the same payoffs given event $E$. Let $\mathcal{F}_{D}$ be the set of acts which are feasible ex ante with respect to decision problem $\mathcal{D}$. Note that $\mathcal{A} \subseteq \mathcal{F}_{D}$, whereas if $\Pi=\{S\}$ is the trivial partition, then $\mathcal{A}=\mathcal{F}_{D}$. An act $f \in \mathcal{F}_{D}$ which is feasible ex ante is optimal if, conditional on each element $E \in \Pi$ of the partition and $f$, it is weakly preferred to any act $g \in \mathcal{A}$.

Definition 1 Act $f \in \mathcal{F}_{D}$ is optimal for decision problem $\mathcal{D}=\{\Pi, \mathcal{A}\}$ if for all $E \in \Pi, f \succsim_{E, f} g$ for all $g \in \mathcal{A}$.

Note that even if optimal $f \in \mathcal{F}_{D}$ does not belong to $\mathcal{A}$, Axiom 5 (Conditional Preference) and ex ante feasibility of $f$ imply that there exists $g \in \mathcal{A}$ with $f={ }_{E} g$ and $f \sim_{E, f} g$, hence it is as if the agent picks $f$ at each cell $E$. We compare two decision problems that differ only in terms of how they partition $S$, by comparing the optimal acts they generate, according to the ex ante preference relation $\succsim$. Decision problem $\mathcal{D}_{1}$ is more valuable than $\mathcal{D}_{2}$ if, for every optimal act for $\mathcal{D}_{2}$ there exists an optimal act for $\mathcal{D}_{1}$ that the agent weakly prefers. It is weakly more valuable if he weakly prefers a convex combination of the two.

Definition 2 Decision problem $\mathcal{D}_{1}=\left\{\Pi_{1}, \mathcal{A}\right\}$ is more valuable than decision problem $\mathcal{D}_{2}=\left\{\Pi_{2}, \mathcal{A}\right\}$ if, whenever act $f \in \mathcal{F}_{\mathcal{D}_{1}}$ is optimal for $\mathcal{D}_{1}$ and act $g \in \mathcal{F}_{\mathcal{D}_{2}}$ is optimal for $\mathcal{D}_{2}$, we have $f \succsim g$. It is weakly more valuable if $a f+(1-a) g \succsim g$ for some $a \in(0,1]$.

Recall that partition $\Pi_{1}$ is finer than partition $\Pi_{2}$ if for every element $E_{2} \in \Pi_{2}$, there exists $E_{1} \in \Pi_{1}$ with $E_{1} \subseteq E_{2}$. Information is (weakly) valuable for $\left\{\succsim_{E, h}\right\}_{E \in \mathcal{E}, h \in \mathcal{F}}$ if a decision problem generated by a finer partition is always (weakly) more valuable. ${ }^{10}$

Definition 3 Information is (weakly) valuable for $\left\{\succsim_{E, h}\right\}_{E \in \mathcal{E}, h \in \mathcal{F}}$ if for all $\mathcal{A} \subseteq \mathcal{F}$ $\left(\mathcal{F}_{+}\right)$, whenever partition $\Pi_{1}$ is finer than partition $\Pi_{2}$, decision problem $\mathcal{D}_{1}=$ $\left\{\Pi_{1}, \mathcal{A}\right\}$ is (weakly) more valuable than decision problem $\mathcal{D}_{2}=\left\{\Pi_{2}, \mathcal{A}\right\}$.

We now show that valuable information is equivalent to Axiom 12 (Information is Valuable), whereas under No Flat Kinks weakly valuable information is equivalent to Axiom 14 (Weak Information is Valuable). Interestingly, we do not need to assume Consequentialism, only Status Quo Bias, which is weaker.

Proposition 1 Suppose Status Quo Bias is satisfied. Then, information is valuable for convex preferences $\left\{\succsim_{E, h}\right\}_{E \in \mathcal{E}, h \in \mathcal{F}}$ if and only if Axiom Information is Valuable is satisfied. Axiom Weak Information is Valuable implies that information is weakly valuable. The converse is true under No Flat Kinks.

10 The definition of weakly valuable information is restricted to feasible sets $\mathcal{A} \subseteq \mathcal{F}_{+}$consisting of strictly positive acts. This simplifies the analysis (as in Hanany and Klibanoff (2009)), because it avoids the multiplicity of supporting hyperplanes at the boundary. 
An interesting question is whether information is always valuable when the partitions contain only unambiguous events. The answer is no. We provide a counterexample with MEU preferences (Gilboa and Schmeidler 1989), adopting the following definition of an unambiguous event, from Nehring (1999) and Ghirardato et al. (2004). ${ }^{11}$ An event $E$ is unambiguous given a set of priors $C$ if for all $p, q \in C$, $p(E)=q(E)$.

Consider a state space with 4 states, $S=\left\{s_{1}, s_{2}, s_{3}, s_{4}\right\}$. The agent has MEU preferences, represented by $U(f)=\min _{p \in C} \sum_{s \in S} u(f(s))$, where $u(x)=x$. The agent's set of priors $C$ is the convex hull of probabilities $(0.4,0.1,0.25,0.25)$ and $(0.1,0.4,0.3,0.2)$. When the agent is informed that event $E \in \Pi$ occurred, he forms beliefs $C_{E}$, using prior-by-prior updating.

We compare two partitions. Partition 1 contains no information, so $\Pi_{1}=\{S\}$, whereas partition 2 is $\Pi_{2}=\left\{\left\{s_{1}, s_{2}\right\},\left\{s_{3}, s_{4}\right\}\right\}$. The three events in these two partitions, $\{S\},\left\{s_{1}, s_{2}\right\},\left\{s_{3}, s_{4}\right\}$, are unambiguous, because all $p \in C$ assign the same probability.

There are two acts, $f=(1,2,-2,3)$ and $g=(1,2,1,-1)$. Let $E=\left\{s_{3}, s_{4}\right\}$. First, note that $U(f)=0.85$ and $U(g)=0.6$. If event $E$ occurs, prior-by-prior updating implies that $C_{E}$ is the convex hull of probabilities $(0.5,0.5)$ and $(0.6,0.4)$. We then have that $U_{E}(f)=U_{E}(g)=0$. This violates the Axiom Value of Information, because $g \succsim_{E} f, f={ }_{E^{c}} g$ but $f \succ g$. In other words, even though all events in the two partitions are unambiguous, the agent with a partition $\Pi_{1}$ would prefer not to obtain the more informative $\Pi_{2}$ ex ante, because the former would yield $f$, whereas with the latter he would choose $g$.

\section{Bayesian updating of subjective beliefs}

RSS define the subjective beliefs at an act $f$ and preference relation $\succsim_{E, h}$ to be the set of all normals (normalized to be probabilities) of the supporting hyperplanes of $f$,

$$
\pi_{E, h}(f)=\left\{p \in \Delta S: \mathbb{E}_{p} g \geq \mathbb{E}_{p} f \text { for all } g \succsim_{E, h} f\right\} .
$$

In Fig. 1, the indifference curve at $f$ has a kink. All the supporting hyperplanes at $f$ are in the shaded area. Set $\pi_{E, h}(f)$ contains their (normalized) normals.

RSS provide two alternative definitions for subjective beliefs and show that all three coincide for strictly positive acts. First, suppose that the agent's endowment is act $f$ and we interpret a probability measure as a set of prices, one for each ArrowDebreu security which pays 1 in a particular state and 0 otherwise. Given preference relation $\succsim_{E, h}$, the subjective beliefs revealed by unwillingness to trade at $f$ contain the measures (prices) for which the agent would be unwilling to trade his endowment,

$$
\pi_{E, h}^{u}(f)=\left\{p \in \Delta S: f \succsim_{E, h} g \text { for all } g \text { such that } \mathbb{E}_{p} g=\mathbb{E}_{p} f\right\}
$$

\footnotetext{
11 See also Amarante and Filiz (2007). In the case of Choquet expected utility preferences, Dominiak and Lefort (2011) study the connection between DC and unambiguous events, whereas Asano and Kojima (2019) axiomatize the Dempster-Shafer and naive Bayes' rules.
} 
Second, let $P$ be a set of measures (prices) such that whenever another act $k$ is unaffordable for every $p \in P$, then there exists a mixture of $k$ with endowment $f$ that the agent would strictly prefer to his endowment. In Fig. 1, there exist some points on the convex combination between $f$ and $k$ that are strictly preferred to $f$. The smallest such $P$ of measures contains the subjective beliefs revealed by willingness to trade at $f$. Formally, let $\mathcal{P}_{E, h}(f)$ denote the collection of all compact, convex sets $P \subseteq \Delta S$ such that if $\mathbb{E}_{p} g>\mathbb{E}_{p} f$ for all $p \in P$, then $\epsilon g+(1-\epsilon) f \succ_{E, h} f$ for sufficiently small $\epsilon>0$. Then, the subjective beliefs revealed by willingness to trade at $f$ are denoted by $\pi_{E, h}^{w}(f)=\bigcap \mathcal{P}_{E, h}(f)$. RSS show that for strictly positive acts $f, \pi_{E, h}(f)=\pi_{E, h}^{u}(f)=\pi_{E, h}^{w}(f)$.

We next define Bayesian updating of subjective beliefs. Note that even though we require that every subjective belief in $\pi(f)$ is updated when $E$ occurs, we allow for the possibility that more subjective beliefs are included given $E$.

Definition 4 The subjective beliefs of $\left\{\succsim_{E, h}\right\}_{E \in \mathcal{E}, h \in \mathcal{F}}$ are updated using Bayes' rule if, for all $E \in \mathcal{E}$ and all $f \in \mathcal{F}_{+}$, if $p \in \pi(f)$ and $p(E)>0$ then $p_{E} \in \pi_{E, f}(f)$.

A convenient and often assumed property of dynamic models with ambiguity is mutual absolute continuity of the priors used to represent preferences. It specifies that all priors put positive probability on the same events. This facilitates the Bayesian updating of all the priors when new information arrives, without worrying how to update priors that assign zero probability on the event. Epstein and Marinacci (2007) characterize mutual absolute continuity in the multiple priors model, using a condition introduced by Kreps (1979). In the following lemma we characterize mutual absolute continuity of the subjective beliefs, using the notion of a weakly null event.

Lemma 2 If event $F \subseteq E \in \mathcal{E}$ is not weakly $\succsim_{E, h}$-null then $p \in \pi_{E, h}^{u}(f)$ implies $p(F)>0$, for all acts $f \in \mathcal{F}$. Conversely, if, for all acts $f \in \mathcal{F}, p \in \pi_{E, h}^{w}(f)$ implies $p(F)>0$, then $F$ is not weakly $\succsim_{E, h}$-null.

Using this lemma, Weak Full Support, which is implied by Strong Monotonicity, ensures that all subjective beliefs $p \in \pi_{E, h}^{u}(f)$ put positive probability at each event $F \in \mathcal{E}$, where $F \subseteq E$.

We now show that Weak Consistency of Implementation (Axiom 13), which is weaker than the second part of DC (Axiom 11), is equivalent to Bayesian updating of subjective beliefs. For the "only if" direction we also need Weak Full Support, which ensures that all subjective beliefs put positive probability on each conditioning event.

Proposition 2 Suppose that convex preferences $\left\{\succsim_{E, h}\right\}_{E \in \mathcal{E}, h \in \mathcal{F}}$ satisfy Weak Full Support. Then, subjective beliefs are updated using Bayes' rule if and only if Weak Consistency of Implementation is satisfied.

\section{Updating rules}

In this section we discuss updating rules using the results of RSS, who characterise the subjective beliefs for various preference models with ambiguity aversion. We 
propose rules which satisfy Bayesian updating of subjective beliefs and therefore Weak Consistency of Implementation. As with the rest of the paper and RSS, the domain of preferences is acts from states to monetary outcomes. In Sect. 6, we discuss how Weak Consistency of Implementation can be extended to a domain of state contingent lotteries. We restrict attention to strictly positive acts $f$, so that $\pi_{h}(f)=\pi_{h}^{u}(f)=$ $\pi_{h}^{w}(f)$. We also assume that whenever $u$ is defined, it is concave, increasing and continuously differentiable.

\subsection{Variational preferences}

Maccheroni et al. (2006a) axiomatize the variational preferences model, which contains as special cases the MEU model of Gilboa and Schmeidler (1989), the multiplier preferences model of Hansen and Sargent (2001) and the mean-variance preferences of Markowitz (1952) and Tobin (1958). The representation of ex ante preferences is

$$
U(f)=\min _{p \in \Delta S}\left\{\int u(f) d p+c(p)\right\},
$$

where $c: \Delta S \rightarrow[0, \infty]$ is a lower semicontinuous convex function, called ambiguity index, with $\min _{p \in \Delta S} c(p)=0$ and $u$ is increasing, concave and differentiable. For simplicity, we assume that if $c(p) \neq+\infty$, then $p(E)>0$ for all $E \in \mathcal{E}$. RSS show that such preferences are convex.

Given event $E \in \mathcal{E}$, act $h \in \mathcal{F}$ and preference relation $\succsim_{E, h}$, the agent's utility at $f$ is $U_{E, h}(f)=\min _{p \in \Delta E}\left\{\int u(f) d p+c_{E, h}(p)\right\}$, where $c_{E, h}$ is the conditional ambiguity index with $\min _{p \in \Delta E} c_{E, h}(p)=0$. Let $M_{E, h}(f)=\underset{p \in \Delta E}{\operatorname{argmin}}\left\{\int u(f) d p+c_{E, h}(p)\right\}$ be the set of minimizing priors realising the utility of $f$.

We define an updating rule by a tuple $\left\{D^{f}, n_{E, f}, k_{E, f}, c_{E, f}\right\}$, for each event $E \in \mathcal{E}$ and act $f$. First, $D^{f} \subseteq \Delta S$ is a convex set such that $M(f) \subseteq D^{f} \cdot{ }^{12}$ Let $D_{E}^{f}$ be the prior by prior updating of $D^{f}$. Second, function $n_{E, f}: D_{E}^{f} \rightarrow \Delta S$ maps each $p \in D_{E}^{f}$ to an unconditional $q \in \Delta S$. Third, let

$$
k_{E, f}=\min _{q \in D_{E}^{f}}\left\{\int u(f) d n_{E, f}(q)+c\left(n_{E, f}(q)\right)-\int u(f) d q\right\} .
$$

The ambiguity index $c_{E, f}: \Delta E \rightarrow[0,+\infty]$ is defined as

$$
c_{E, f}(p)=\int u(f) d n_{E, f}(p)+c\left(n_{E, f}(p)\right)-\int u(f) d p-k_{E, f},
$$

if $p \in D_{E}^{f}$, otherwise $c_{E, f}(p)=+\infty$. Subtracting $k_{E, f}$ ensures that $c_{E, f}(p) \in$ $[0,+\infty]$ and $c_{E, f}(p)=0$ for some $p$. In order to ensure that $c_{E, f}$ is convex, continuous and subjective beliefs are updated, we consider two specific functions $n_{E, f}$.

\footnotetext{
12 Note that $M(f)$ is convex.
} 
For the first updating rule, fix measure $r \in M(f)$ with $r(E)>0$ and let $D^{f}$ such that $p \in D^{f}$ implies $c(p) \neq+\infty .{ }^{13}$ Set $n_{E, f}(p)=r$ for all $p \in D_{E}^{f}$.

The second rule applies only in the case where $M(f)=\{r\}$ is a singleton and $r(E)>0$. Set $D^{f}=\Delta S$ and let $n_{E, f}(p)=p \otimes^{E} r$ for all $p \in \Delta E$, where $p \otimes^{E} r \in \Delta S$ is such that $p \otimes^{E} r(F)=r(E) p(F)+r\left(F \cap E^{c}\right)$ for all events $F$. Then, in $p \otimes{ }^{E} r$ the choice of $p$ determines all probabilities given $E$ whereas $r$ determines all other probabilities.

We next show that these two rules generate variational preferences that satisfy Bayesian updating of subjective beliefs and Weak Consistency of Implementation (Axiom 13), although DC (Axioms 11 and 12) is violated in general. Interestingly, for the first rule, if we set $D^{f}=D$ to be the set of all measures with $c(p) \neq+\infty$, for all $f \in \mathcal{F}$, so that it is independent of $f$, then Consistency of Implementation (Axiom 11) is satisfied. If $D^{f}=M(f)$ for all $f \in \mathcal{F}$, then Information is Valuable (Axiom 12) is satisfied. Hence, the largest possible set of prior by prior updating satisfies Consistency of Implementation, whereas the smallest possible set satisfies Information is Valuable. Any set which is in between, satisfies Weak Consistency of Implementation.

Lemma 3 Suppose that $\succsim$ is a variational preference. Then, both updating rules

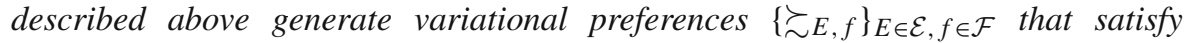
Bayesian updating of subjective beliefs and Weak Consistency of Implementation. Moreover, in the first rule if $D^{f}=D$ for all $f \in \mathcal{F}$ and $c(p) \neq+\infty$ implies $p \in D$, then Consistency of Implementation is satisfied. If $D^{f}=M(f)$ for all $f \in \mathcal{F}$, then Axiom Information is Valuable is satisfied. However, in general both rules violate Consistency of Implementation and Information is Valuable.

\subsection{Confidence preferences}

Chateauneuf and Faro (2009) axiomatize a class of preferences where ambiguity is measured by a confidence function $\phi: \Delta S \rightarrow[0,1]$, with $\phi(p)=1$ meaning full confidence in $p$. The set of full confidence measures is nonempty and $\phi$ is upper semicontinuous and quasiconcave. Preferences are represented by

$$
U(f)=\min _{p \in L_{a}} \frac{1}{\phi(p)} \mathbb{E}_{p} u(f),
$$

where $L_{a}=\{q \in \Delta S: \phi(q) \geq a\}$ is a set of measures with confidence of at least $a>0$. Define $M(f)=\underset{p \in L_{a}}{\arg \min }\left\{\frac{1}{\phi(p)} \mathbb{E}_{p} u(f)\right\}$ for each $f \in \mathcal{F}$. For simplicity, we assume that if $p \in L_{a}$ then $p(E)>0$ for all $E \in \mathcal{E}$. Hence, Weak Full Support (Axiom 8) is satisfied.

Consider the following updating rule. Fix measure $r \in M(h)$ with $r(E)>0$ and convex set $D^{h}$ such that $M(h) \subseteq D^{h} \subseteq L_{a}$. Let $D_{E}^{h}$ be the prior by prior updating of

\footnotetext{
13 Note that if there does not exist $r \in M(h)$ with $r(E)>0$, Bayesian updating of subjective beliefs is trivially satisfied.
} 
$D^{h}$, given $E \in \mathcal{E}$. Let $k_{E, h}=\max _{q \in D_{E}} \phi(r) \frac{\mathbb{E}_{q} u(h)}{\mathbb{E}_{r} u(h)}$. For each $p \in \Delta E$, define

$$
\phi_{E, h}(p)=\frac{\phi(r)}{k_{E, h}} \frac{\mathbb{E}_{p} u(h)}{\mathbb{E}_{r} u(h)}
$$

if $p \in D_{E}$, otherwise $\phi_{E, h}(p)=0$. Setting $a_{E, h}=\min _{p \in D_{E}} \phi_{E, h}(p)$, we have that $D_{E}^{h}=L_{a_{E, h}}$ and $U_{E, h}(f)=\min _{p \in L_{a_{E, h}}} \frac{1}{\phi_{E, h}(p)} \mathbb{E}_{p} u(f)$.

We now show that this updating rule satisfies Weak Consistency of Implementation. As with the first updating rule on variational preferences, the largest possible prior by prior updating set additionally satisfies Consistency of Implementation, whereas the smallest satisfies Information is Valuable. However, if $D^{h}$ is a strict subset of $L_{a}$, then Consistency of Implementation may be violated.

Lemma 4 Suppose that $\succsim$ is a confidence preference. Then, the updating rule described above generates confidence preferences $\left\{\succsim_{E, h}\right\}_{E \in \mathcal{E}, h \in \mathcal{F}}$ that satisfy Bayesian updating of subjective beliefs and Weak Consistency of Implementation. Moreover, if $D^{f}=L_{a}$ for all $f \in \mathcal{F}$, then Consistency of Implementation is satisfied. If $D^{f}=M(f)$ for all $f \in \mathcal{F}$, then Information is Valuable is satisfied. If $D^{h}$ is a strict subset of $L_{a}$, then Consistency of Implementation may be violated.

\section{Discussion}

Before discussing the literature, we compare our approach with Ghirardato et al. (2004) and Hanany and Klibanoff $(2007,2009)$, which are closely related. One notable difference is that they use the larger domain of Anscombe and Aumann (1963). That is, preferences are over acts $f: S \rightarrow X$, where $X$ is the set of simple lotteries over a set of consequences $Z$. In order to compare our approach with theirs, we assume that $Z=\mathbb{R}_{+}$. Let $\mathcal{F}_{A A}$ be the set of such acts. Our definition of revealed preference in the larger domain $\mathcal{F}_{A A}$ is extended as follows. For all $f, g \in \mathcal{F}_{A A}, f \succsim_{\mathcal{A} \mathcal{A}} g$ if $f \succsim a g+(1-a) f$ for all $a \in[0,1]$. Note that the mixed act $a g+(1-a) f$ has a different interpretation in this section. At state $s$, it gives lottery $g(s)$ with probability $a$ and lottery $f(s)$ with probability $1-a$. In the previous sections of the paper, where the restricted domain $\mathcal{F}$ applies, at state $s$ act $g \succsim a f+(1-a) f$ pays the expected payoff $a g(s)+(1-a) f(s) \in Z$ for sure.

\subsection{Unambiguous preferences}

Ghirardato et al. (2004) define $f$ to be unambiguously preferred to $g$, written $f \succsim_{G M M}^{*}$ $g$, if mixing with any other act $h$ does not reverse preferences: $\alpha f+(1-\alpha) h \succsim$ $\alpha g+(1-\alpha) h$ for all acts $f, g, h \in \mathcal{F}_{A A}$ and $\alpha \in(0,1]$. Our extended notion of revealed preference (given Consequentialism) requires mixing only with $h=f$, so that $f \succsim_{\mathcal{A} \mathcal{A}}^{*} g$ if $f \succsim a g+(1-a) f$ for all $f, g, \in \mathcal{F}_{\mathcal{A} \mathcal{A}}$ and $a \in[0,1]$, hence it is weaker. That is, $f \succsim_{G M M}^{*} g$ implies $f \succsim_{\mathcal{A} \mathcal{A}}^{*} g$ for all $f, g \in \mathcal{F}_{A A}$. 
Although the representation Ghirardato et al. (2004) is in a setting which satisfies the Certainty Independence Axiom of Gilboa and Schmeidler (1989), Cerreia-Vioglio et al. (2011) show that it holds more generally for uncertainty averse preferences. In particular, they show that $f \succsim_{G M M}^{*} g$ if and only if $\int u(f) d p \geq \int u(g) d p$ for all $p \in C$, where $C=\operatorname{cl}\left(\operatorname{co}\left(\bigcup_{f \in \mathcal{F}} \pi_{0}^{u}(f)\right)\right)$ and $\pi_{0}^{u}(f)=\{p \in \Delta S: f \succsim$ $g$ for all $g$ such that $\left.\mathbb{E}_{p} u(f) \geq \mathbb{E}_{p} u(g)\right\}$ is the set of subjective beliefs evaluated at $u(f)$, rather than at $f$, as is $\pi^{u}(f)$.

Ghirardato et al. (2008) provide a dynamic version of Ghirardato et al. (2004), assuming Conditional Preference, Consequentialism and DC on $\succsim_{G M M}^{*}$, so that $f \succsim_{G M M, E}^{*} g$ if and only if $f \succsim_{G M M}^{*} g$, for all acts $f, g$ with $f=E^{c} g$. They show that Bayesian updating of all beliefs in $C$ is equivalent to $\succsim_{G M M}^{*}$ satisfying DC.

\subsection{Uncertainty averse preferences}

Hanany and Klibanoff (2007) relax Consequentialism, allowing for preferences conditional on an event to also depend on the feasible set $B$ of acts and on the act that was chosen ex ante. They show in the MEU model that a weakening of DC is equivalent to Bayesian updating of a subset of the unconditional beliefs. Moreover, their model allows for both Ellsberg type behavior and non-reversal of preferences when information is revealed, hence DC is satisfied. Hanany and Klibanoff (2009) generalise their approach to models that satisfy the uncertainty aversion axiom Schmeidler (1989), showing that their Dynamic Consistency Axiom is equivalent to having at least one measure which supports both the conditional indifference curve at the chosen act and its conditional optimality.

To explain their approach, let $\mathcal{Q}$ be the set of all quadruples $(\succsim, E, f, B$ ), where non-null $E$ is the conditioning event and act $f \in \mathcal{F}_{A A}$ is optimally chosen from a convex feasible set $B \in \mathcal{B}(f \succsim g$ for all $g \in B)$, before the realization of $E$, where $\mathcal{B}$ is the set of all convex and compact sets. Let $\mathcal{D} \subseteq \mathcal{Q}$ be a domain if $(\succsim, E, f, B) \in \mathcal{D}$ implies $\left(\succsim, E, f^{\prime}, B^{\prime}\right) \in \mathcal{D}$ for each $f^{\prime}, B^{\prime}$ such that $\left(\succsim, E, f^{\prime}, B^{\prime}\right) \in \mathcal{Q}$ and $u \circ f^{\prime}$ is the unique maximizer of $V$ (which represents preferences) over $u \circ B^{\prime}$. Their Dynamic Consistency axiom is the following.

Axiom $15\left(D C_{H K}\right)$ For any $(\succsim, E, f, B) \in \mathcal{D}$, if $g \in B$ with $f={ }_{E^{c}} g$, then $f \succsim_{E, f, B}$ $g$.

The main difference of their approach from ours is that preferences depend not only on the conditioning event $E$ and act $f$, but also on the feasible set $B$. The current paper additionally imposes that $\succsim_{E, f, B}=\succsim_{E, f, B^{\prime}}$ for all convex feasible sets $B$ and $B^{\prime}$. To state the difference formally, we extend Weak Consistency of Implementation (Axiom 13) to the bigger $\mathcal{F}_{A A}$ domain. ${ }^{14}$

Axiom $16\left(W C O I_{A A}\right)$ For all acts $f, g \in \mathcal{F}_{A A}$ and events $E \in \mathcal{E}$, if $f \succsim_{\mathcal{A} \mathcal{A}}^{*} g$ and $f={ }_{E^{c}} g$ then $f \succsim_{E, f} g$.

\footnotetext{
14 For simplicity, we assume that all acts in $\mathcal{F}_{A A}$ are strictly positive in utility space (the interior acts in Hanany and Klibanoff (2009)).
} 
We now show that the two axioms are equivalent, given that conditional preferences do not depend on the feasible set $B$.

Lemma 5 Suppose $\succsim_{E, f, B}=\succsim_{E, f, B^{\prime}}$ for all convex feasible sets $B$ and $B^{\prime}$. Then, $D C_{H K}$ is equivalent to $W C O I_{A A}$.

In general, however, whenever $\succsim_{E, f, B} \neq \succsim_{E, f, B^{\prime}}$ is allowed, $W C O I_{A A}$ is stronger than $D C_{H K}$, as it requires that $\succsim_{F, f, B}=\succsim_{F, f, B^{\prime}}$ for all $B, B^{\prime}$. The characterizations in Hanany and Klibanoff $(2007,2009)$ are provided for the general case, except for the smooth ambiguity model, where there is a unique subjective belief at each $f$ and preferences do not depend on $B$.

We now show that, in the case of variational preferences where subjective beliefs are not unique and $\succsim_{F, f, B} \neq \succsim_{F, f, B^{\prime}}$, a rule proposed by Hanany and Klibanoff (2009) which satisfies $D C_{H K}$ implies that at least one subjective belief is updated, but not all. This means that Bayesian updating of subjective beliefs and Weak Consistency of Implementation fail.

Fix $r \in M(f) \cap Q^{E, f, B}$, where $Q^{E, f, B}$ is the set of measures which, when conditioned on $E$, support the conditional optimality of $f$ in $B .{ }^{15}$ The ambiguity index of their proposed updating rule is

$$
c_{E, f, B}(p)=\frac{1}{r(E)}\left[c\left(p \otimes^{E} r\right)-\min _{q \in \Delta E} c\left(q \otimes^{E} r\right)\right],
$$

where $p \otimes^{E} r \in \Delta S$ is such that $p \otimes^{E} r(F)=r(E) p(F)+r\left(F \cap E^{c}\right)$ for all events $F$. Note that for all $p \in \Delta E, \int u(f) d p+c_{E, f, B}(p) \geq \int u(f) d r_{E}+c_{E, f, B}\left(r_{E}\right)$ if and only if $r(E) \int u(f) d p+c\left(p \otimes^{E} r\right) \geq r(E) \int u(f) d r_{E}+c(r)$, which is true because $\int u(f) d p \otimes^{E} r=r(E) \int u(f) d p+\int_{E^{c}} u(f) d r$ and $r \in M(f)$. Using the proof of Lemma 8, we can show that the subjective belief generated by $r$ is updated.

If, however, $q \in M(f)$ and $q \neq r$, then it is not necessarily the case that $\int u(f) d p+c_{E, f, B}(p) \geq \int u(f) d q_{E}+c_{E, f, B}\left(q_{E}\right)$ for all $p \in \Delta E$, which means that the subjective belief generated by $q$ is not updated. The reason is that we need $r(E) \int u(f) d p+c\left(p \otimes^{E} r\right) \geq r(E) \int u(f) d q_{E}+c\left(q_{E} \otimes^{E} r\right)$, which is equivalent to $\int u(f) d p \otimes^{E} r+c\left(p \otimes^{E} r\right) \geq \int u(f) d q_{E} \otimes^{E} r+c\left(q_{E} \otimes^{E} r\right)$, however if $q_{E} \otimes{ }^{E} r \notin M(f)$ this may not be true. Hence, not all subjective beliefs are updated and Axiom 13 is violated.

To provide a numerical example, consider the setting of Sect. 1.1 with MEU ex ante preferences, $u(x)=x$ and $C$ being the convex hull of the following three measures: $p_{1}=(0.24,0.33,0.43), p_{2}=(0.34,0.2,0.46)$ and $p_{3}=(0.21,0.68,0.11)$. Let $f=$ $x \in \mathbb{R}$ be a constant act and note that $M(f)=C$. Pick $r=p_{1}$ and define feasible set $B$ such that $f \in B$ if $\mathbb{E}_{r} x \geq \mathbb{E}_{r} f$. Then, $p_{1} \in M(f) \cap Q^{E, f, B}$. If we pick $p_{1}$ to generate $c_{E, x, B}$, using (3), then we have that $p_{2 E} \otimes^{E} p_{1}=\{0.3589,0.2111,0.4300\} \notin C$. This means that $c_{E, x, B}\left(p_{2 E}\right)=+\infty$ and $p_{2 E} \notin M_{E, x}(x)$, hence not all subjective beliefs are updated.

Finally, we show that in the smooth ambiguity model (Maccheroni et al. 2006a), where subjective beliefs are unique at each $f$, Hanany and Klibanoff (2009) propose

\footnotetext{
15 The formal definition of $Q^{E, f, B}$ is in Hanany and Klibanoff (2009).
} 
a rule which satisfies Bayesian updating of subjective beliefs. An act $f$ is weakly preferred to $g$ if and only if $\mathbb{E}_{\mu} \phi\left(\mathbb{E}_{p} u \circ f\right) \geq \mathbb{E}_{\mu} \phi\left(\mathbb{E}_{p} u \circ g\right)$, where $\phi: \mathbb{R} \rightarrow \mathbb{R}$ is an increasing transformation called the ambiguity attitude and $\mu$ is a subjective probability over the set of probability measures $p \in \Delta S$ that the agent considers relevant for his problem. For simplicity, we assume that $\mu$ has finite support and that $u$ and $\phi$ are concave, increasing and differentiable, so that preferences are convex. We also assume Axiom 8, which implies that for each $E \in p$, there exists $p \in \operatorname{supp}(\mu)$ with $p(E)>0$.

Hanany and Klibanoff (2009) propose the smooth rule, which specifies that

$$
\mu_{E, f}(p)=\frac{\mu(p) p(E) \frac{\phi^{\prime}\left(\mathbb{E}_{p}(u \circ f)\right)}{\phi^{\prime}\left(\mathbb{E}_{p_{E}}(u \circ f)\right)}}{\sum_{\hat{p} \in \Delta S} \mu(\hat{p}) \hat{p}(E) \frac{\phi^{\prime}\left(\mathbb{E}_{\hat{p}}(u \circ f)\right)}{\phi^{\prime}\left(\mathbb{E}_{\hat{p}_{E}}(u \circ f)\right)}}
$$

if $p(E)>0$ and 0 otherwise.

The smooth rule satisfies the following condition, which Hanany and Klibanoff (2009) show that it characterizes $D C_{H K}$ :

$$
\frac{\mathbb{E}_{\mu_{E, f}}\left[\phi^{\prime}\left(\mathbb{E}_{p_{E}} u \circ f\right) p_{E}(s)\right]}{\mathbb{E}_{\mu_{E, f}}\left[\phi^{\prime}\left(\mathbb{E}_{p_{E}} u \circ f\right)\right]}=\frac{\mathbb{E}_{\mu}\left[\phi^{\prime}\left(\mathbb{E}_{p} u \circ f\right) p(s)\right]}{\mathbb{E}_{\mu}\left[\phi^{\prime}\left(\mathbb{E}_{p} u \circ f\right) p(E)\right]}
$$

for all $f \in \mathcal{F}, s \in E$ and $p \in \operatorname{supp}(\mu)$ with $p(E)>0 .{ }^{16}$ We show that (4) satisfies Bayesian updating of subjective beliefs.

Lemma 6 There is Bayesian updating of subjective beliefs for any rule satisfying (4).

\subsection{Related literature}

Cerreia-Vioglio et al. (2011) study general convex preferences which satisfy the uncertainty aversion axiom of Schmeidler (1989). Epstein and Schneider (2003), Maccheroni et al. (2006b) and Klibanoff et al. (2009) use DC in order to provide recursive representations for the static models of Gilboa and Schmeidler (1989), Maccheroni et al. (2006a) and Klibanoff et al. (2005), respectively. Several other papers employ DC, such as Eichberger and Kelsey (1996), Eichberger et al. (2005, 2016), Takashi (2005) Sarin and Wakker (1998), Wang (2003), Hayashi and Miao (2011) and Vergopoulos (2011).

Siniscalchi (2011) drops DC completely and replaces it with Consistent Planning, which specifies that the agent adjusts his actions today in order to restrict his future self's choices, because he recognises that his preferences will change tomorrow. This approach accommodates Ellsberg but information is not valuable, unless the agent can exogenously commit.

Bayesian updating of priors is suggested or characterized by Jaffray (1992, 1994), Fagin and Halpern (1991), Wasserman and Kadane (1990), Walley (1991), Epstein and

\footnotetext{
$\overline{16}$ Note that we state the condition on $\mathcal{F}$, whereas Hanany and Klibanoff (2009) state it on $\mathcal{F}_{\mathcal{A} \mathcal{A}}$.
} 
Schneider (2003), Sarin and Wakker (1998), Pires (2002), Siniscalchi (2001), Wang (2003) and Faro and Lefort (2019). Ghirardato et al. (2008) characterize the Bayesian updating of a set of beliefs which are used to represent the unambiguous preference relation (Ghirardato et al. 2004), which is incomplete. Gilboa and Schmeidler (1993) analyze maximum likelihod updating, whereas Dempster (1968) and Shafer (1976) suggest the Dempster-Shafer updating rule. Epstein (2006) provides an axiomatic model of non-Bayesian updating.

Another property of Consequentialism (which we call Conditional Preference in this paper and assume it throughout) is that conditional on an event $E$, the agent only cares about what the act prescribes inside $E$. Dominiak et al. (2012) show experimentally that subjects are more prone to violating DC than this property.

RSS identify the subjective beliefs generated by a large number of models of ambiguity aversion, based on an idea of Yaari (1969), making our approach very general. These models are the convex Choquet model of Schmeidler (1989), the multiple priors model of Gilboa and Schmeidler (1989), the variational preferences model of Maccheroni et al. (2006a), the multiplier model of Hansen and Sargent (2001), the smooth second-order prior models of Klibanoff et al. (2005) and Nau (2006), the confidence preferences model of Chateauneuf and Faro (2009) and the second-order expected utility model of Ergin and Gul (2009). ${ }^{17}$ Ghirardato and Siniscalchi (2018) extend the approach of RSS to non convex preferences in order to characterize betting in terms of disjoint beliefs.

In a setting with preferences over lotteries, Wakker (1988) shows that if Independence is violated, the value of information is not always positive. Independence is related to DC and, under some conditions (e.g. English auctions), it is equivalent (Karni and Safra 1986). Grant et al. (2000) provide necessary and sufficient conditions for a weakly dynamically consistent agent to always prefer more information. Our approach differs from theirs in two respects. First, they adopt the definition of "more information" suggested by Blackwell (1951), whereas we adopt the definition of a finer partition. ${ }^{18}$ Second, they adopt a different weakening of DC, due to Machina (1989), which requires that an agent conforms to what he would have chosen ex ante only if he were able to commit. Snow (2010) examines the value of information in the special case where it either reduces or eliminates ambiguity, using the model of Klibanoff et al. (2005). Li (2020) studies the link between ambiguity attitudes and aversion to receiving information when one is completely uninformed. Galanis (2015, 2016) examines the value of information in environments with unawareness, where DC is violated. Finally, Galanis and Kotronis (Forthcoming) and Galanis et al. (2019) study under which conditions financial markets aggregation information, when DC is violated.

Open Access This article is licensed under a Creative Commons Attribution 4.0 International License, which permits use, sharing, adaptation, distribution and reproduction in any medium or format, as long as you give appropriate credit to the original author(s) and the source, provide a link to the Creative Commons licence,

\footnotetext{
17 Note that RSS adopts a domain of preferences over monetary acts, whereas these models allow for more general domains.

18 The two definitions are closely related, as shown by Green and Stokey (1978).
} 
and indicate if changes were made. The images or other third party material in this article are included in the article's Creative Commons licence, unless indicated otherwise in a credit line to the material. If material is not included in the article's Creative Commons licence and your intended use is not permitted by statutory regulation or exceeds the permitted use, you will need to obtain permission directly from the copyright holder. To view a copy of this licence, visit http://creativecommons.org/licenses/by/4.0/.

\section{A Appendix}

\section{A.1 Revealed acts}

In order to prove our results, we introduce the notion of revealed acts and connect it with preference relation $\succsim^{*}$. We have interpreted $\pi_{E, h}^{u}(f)$ as the set of (normalized) Arrow-Debreu prices for which the agent with preferences $\succsim_{E, h}$, endowed with $f$, would have zero net demand. The "dual" of $\pi_{E, h}^{u}(f)$ is the set of acts for which $f$ is revealed preferred to them. In particular, if $\mathbb{E}_{p} f \geq \mathbb{E}_{p} g$ then act $g$ is affordable given normalized price $p$ and endowment $f$. If $p \in \pi_{E, h}^{u}(f)$, then from Axiom 3 we have $f \succsim_{E, h} g$, which means that $f$ is revealed preferred to $g$. Formally, for act $f$ and event $E \in \mathcal{E}$, let $\mathcal{R}_{E, h}^{u}(f)$ be the set of acts such that $f$ is revealed preferred to them given preferences $\succsim E, h$,

$$
\mathcal{R}_{E, h}^{u}(f)=\left\{g \in \mathcal{F}: \mathbb{E}_{p} f \geq \mathbb{E}_{p} g \text { for some } p \in \pi_{E, h}^{u}(f)\right\} .
$$

If we use beliefs $\pi_{E, h}^{w}(f)$, instead of $\pi_{E, h}^{u}(f)$, we get

$$
\mathcal{R}_{E, h}^{w}(f)=\left\{g \in \mathcal{F}: \mathbb{E}_{p} f \geq \mathbb{E}_{p} g \text { for some } p \in \pi_{E, h}^{w}(f)\right\} .
$$

The connection between $\mathcal{R}_{E, h}^{w}, \mathcal{R}_{E, h}^{u}$ and $\succsim_{E, h}^{*}$ is given by the following Lemma.

Lemma 7 For all $f, g, h \in \mathcal{F}, f \succsim_{E, h}^{*} g$ implies $g \in \mathcal{R}_{E, h}^{w}(f)$ and $g \in \mathcal{R}_{E, h}^{u}(f)$ implies $f \succsim_{E, h}^{*} g$.

Proof Suppose $f \succsim_{E, h}^{*} g$, then $f \succsim_{E, h} a g+(1-a) f$ for all $a \in[0,1]$. Suppose $g \notin \mathcal{R}_{E, h}^{w}(f)$, so that $\mathbb{E}_{p} f<\mathbb{E}_{p} g$ for all $p \in \pi_{E, h}^{w}(f)$. Because $\pi_{E, h}^{w}(f)$ is a compact and convex set, by definition we have $\epsilon g+(1-\epsilon) f \succ_{E, h} f$ for sufficiently small $\epsilon>0$, a contradiction. Conversely, suppose $g \in \mathcal{R}_{E, h}^{u}(f)$, so that $\mathbb{E}_{p} f \geq \mathbb{E}_{p} g$ for some $p \in \pi_{E, h}^{u}(f)$. This implies that for all $a \in[0,1], \mathbb{E}_{p} f \geq a \mathbb{E}_{p} g+(1-a) \mathbb{E}_{p} f$. Axiom 3 and the definition of $\pi_{E, h}^{u}(f)$ imply $f \succsim_{E, h} a g+(1-a) f$, hence $f \succsim_{E, h}^{*} g$.

Proposition 1 in RSS shows that $\pi_{E, h}(f)=\pi_{E, h}^{u}(f)=\pi_{E, h}^{w}(f)$ for all strictly positive acts $f \in \mathcal{F}_{+}$. Hence, $\mathcal{R}_{E, h}^{u}(f)=\mathcal{R}_{E, h}^{w}(f) \equiv \mathcal{R}_{E, h}(f)$. Consider the following two axioms, which are equivalent of the two axioms of weak DC, Axioms 13 and 14.

Axiom 17 (Weak Consistency of Implementation) For all acts $f \in \mathcal{F}_{+}, g \in \mathcal{F}$ and events $E \in \mathcal{E}$, if $g \in \mathcal{R}^{u}(f)$ and $f=E_{E^{c}} g$ then $f \succsim_{E, f} g$. 
Axiom 18 (Weak Information is Valuable) For all acts $g \in \mathcal{F}_{+}, f \in \mathcal{F}$ and events $E \in \mathcal{E}$, if $f \succsim_{E, f} g, f=E_{E^{c}} g$ and $g \succ f$ then $f \notin \mathcal{R}^{w}(g)$.

We therefore have the following Corollary.

Corollary 1 For all $f \in \mathcal{F}_{+}, g, h \in \mathcal{F}, f \succsim_{E, h}^{*} g$ if and only if $g \in \mathcal{R}_{E, h}(f)$. Hence, Axiom 13 is equivalent to Axiom 17 and Axiom 14 is equivalent to 18.

\section{A.2 Proofs}

Proof of Lemma 1 Suppose Axioms 10, 12 and let $f \succsim g$ with $f=E^{c} g$. From Axiom 6, for all $a \in(0,1), a f+(1-a) g \succ g$. Axiom 12 implies $a f+(1-a) g \succ_{E, g} g$ for all $a \in(0,1)$. Because $\lim _{a \rightarrow 1}[a f+(1-a) g]=f$, Axiom 2 implies $f \succsim_{E, g} g$. Axiom 10 implies $f \succsim_{E, f} g$.

Conversely, suppose Axioms 9, 11 and let $f \succsim_{E, f} g$ with $f={ }_{E^{c}} g$. From Axiom 6, for all $a \in(0,1), a f+(1-a) g \succ_{E, f} g$. Axiom 9 implies $a f+(1-a) g \succ_{E, g} g$. Axiom 11 implies $a f+(1-a) g \succ g$ for all $a \in(0,1)$. Because $\lim _{a \rightarrow 1}[a f+(1-a) g]=f$, Axiom 2 implies $f \succsim g$.

For the second claim, using Corollary 1 suppose Axioms 10, 14, $g \in \mathcal{R}(f), f=E^{c}$ $g$ and $g \succ_{E, f} f$. Axiom 10 implies $g \succsim_{E, g} f$. If $f \succ g$ then Axiom 14 and Corollary 1 imply $g \notin \mathcal{R}(f)$, a contradiction. Suppose $g \succsim f$. From Axiom 6 we have $a f+(1-a) g \succ f$ for all $a \in(0,1)$. This implies that $\mathbb{E}_{p}(a f+(1-a) g)>\mathbb{E}_{p} f$ for all $p \in \pi(f)$. But then $\mathbb{E}_{p} g>\mathbb{E}_{p} f$ for all $p \in \pi(f)$ and $g \notin \mathcal{R}(f)$, a contradiction.

Conversely, suppose Axioms 9, 13, $f \succsim_{E, f} g, f=E_{E^{c}} g$ and $g \succ f$. From Axiom 6, we have that $a f+(1-a) g \succ_{E, f} g$ for all $a \in(0,1)$. Axiom 9 implies $a f+(1-a) g \succ_{E, g} g$. Axiom 13 and Corollary 1 imply that $a f+(1-a) g \notin \mathcal{R}(g)$, hence $\mathbb{E}_{p}(a f+(1-a) g)>\mathbb{E}_{p} g$ for all $p \in \pi(g)$. But this implies that $\mathbb{E}_{p} f>\mathbb{E}_{p} g$ for all $p \in \pi(g)$, hence $f \notin \mathcal{R}(g)$ and $g \mathcal{L}^{*} f$.

Proof of Lemma 2 Suppose that for some $p \in \pi_{E, h}^{u}(f)$ we have $p(F)=0$. Take any $g$ such that $g={ }_{F^{c}} f$. Because $\mathbb{E}_{p} f=\mathbb{E}_{p} g$, we have $f \succsim_{E, h} g$, implying that $F$ is weakly $\succsim_{E, h}$-null. Conversely, suppose that for all acts $f, p \in \pi_{E, h}^{w}(f)$ implies $p(F)>0$. Suppose there exists act $f$ such that for all $g$ with $f=F^{c} g, f \succsim_{E, h} g$. Let $k>0$ and define act $g$ such that $g(s)=f(s)+k$ if $s \in F$ and $g(s)=f(s)$ otherwise. Then, for all $p \in \pi_{E, h}^{w}(f), \mathbb{E}_{p} g>\mathbb{E}_{p} f$. From the definition of $\pi_{E, h}^{w}(f)$, there exists small enough $\epsilon>0$, such that $g^{\prime}=\epsilon g+(1-\epsilon) f$ and $g^{\prime} \succ f$. Because $f=F_{F} g^{\prime}$, we have a contradiction.

Proof of Proposition 1 Recall that convex preferences satisfy Axioms 1 through 5. Suppose that for any event $E \in \mathcal{E}$ and all acts $f, g \in \mathcal{F}, f \succsim_{E, f} g$ and $f={ }_{E^{c}} g$ implies $f \succsim g$. Consider decision problems $\mathcal{D}_{1}=\left\{\Pi_{1}, \mathcal{A}\right\}$ and $\mathcal{D}_{2}=\left\{\Pi_{2}, \mathcal{A}\right\}$, where $\Pi_{1}, \Pi_{2} \subseteq \mathcal{E}$ are partitions of $S$ and $\Pi_{1}$ is finer than $\Pi_{2}$. Let act $f \in \mathcal{F}_{\mathcal{D}_{1}}$ be optimal for $\mathcal{D}_{1}$ and act $g \in \mathcal{F}_{\mathcal{D}_{2}}$ be optimal for $\mathcal{D}_{2}$. Since $\Pi_{2}$ is coarser than $\Pi_{1}, \mathcal{F}_{\mathcal{D}_{2}} \subseteq \mathcal{F}_{\mathcal{D}_{1}}$ and $g \in \mathcal{F}_{\mathcal{D}_{1}}$. This means that, for all $E \in \Pi_{1}, f \succsim_{E, f} g$.

Enumerate the partition cells of $\Pi_{1}=\left\{E_{1}, \ldots, E_{n}\right\}$. If $n=1$ then $\Pi_{1}=\{S\}$ is the uninformative partition and the result is immediate, so suppose that $n \geq 2$. For cell 
$1 \leq k \leq n$ define act $h_{k}$ as follows. Let $h_{k}(s)=f(s)$ if $s \in E_{j}$, where $1 \leq j \leq k$, and $h_{k}(s)=g(s)$ otherwise. Note that $h_{n}=f$ and let $h_{0}=g$. From Axiom 5 we have that, for each $1 \leq k \leq n, f \succsim_{E_{k}, f} g$ implies $h_{k} \succsim_{E_{k}, f} h_{k-1}$. From Axiom 10 we have $h_{k} \succsim E_{k}, h_{k} h_{k-1}$. Applying Axiom 12 we have $h_{k} \succsim h_{k-1}$, for each $1 \leq k \leq n$. Axiom 1 implies that $f \succsim g$.

Conversely, suppose that Axiom 12 is false, so that for some event $E \in \mathcal{E}$ and acts $f, g \in \mathcal{F}$, we have $f \succsim_{E, f} g$ and $f=E_{E^{c}} g$ but $g \succ f$. Consider partitions $\Pi_{1}=\left\{E, E^{c}\right\}$ and $\Pi_{2}=\{S\}$. Let $\mathcal{A}=\{f, g\}$. Then, $g$ is optimal for decision problem $\mathcal{D}_{2}=\left\{\Pi_{2}, \mathcal{A}\right\}$. Because $f={ }_{E^{c}} g$, Axiom 5 implies that $f \succsim_{E^{c}, f} g$. Since $f \succsim_{E, f} g$, we have that $f$ is optimal for decision problem $\mathcal{D}_{1}=\left\{\Pi_{1}, \mathcal{A}\right\}$. Because $g \succ f, \mathcal{D}_{1}$ is not more valuable than $\mathcal{D}_{2}$, hence information is not valuable.

For the second claim, suppose Axiom 14, which from Corollary 1 implies Axiom 18. Consider the same decision problems as in the first paragraph of this proof (where now $\left.\mathcal{A} \subseteq \mathcal{F}_{+}\right)$and let act $f \in \mathcal{F}_{\mathcal{D}_{1}}$ be optimal for $\mathcal{D}_{1}$ and act $g \in \mathcal{F}_{\mathcal{D}_{2}}$ be optimal for $\mathcal{D}_{2}$. Since $\Pi_{2}$ is coarser than $\Pi_{1}, \mathcal{F}_{\mathcal{D}_{2}} \subseteq \mathcal{F}_{\mathcal{D}_{1}}$ and $g \in \mathcal{F}_{\mathcal{D}_{1}}$. This means that, for all $E \in \Pi_{1}, f \succsim_{E, f} g$. If $f \succsim g$ then for $a=1$ we have $a f+(1-a) g \succsim g$ and information is weakly valuable.

Suppose $g \succ f$. For each $E \in \Pi_{1}$, Axiom 5 and $f \succsim_{E, f} g$ imply $f E g \succsim_{E, f} g$. Axiom 10 implies that $f E g \succsim_{E, f E g} g$. From Axiom 18, either $f E g \succsim g$ or $g \succ f E g$ and $f E g \notin \mathcal{R}(g)$, which implies that $\mathbb{E}_{p} f E g \geq \mathbb{E}_{p} g$ for all $p \in \pi(g)$. Because $\Pi_{1}$ is a partition of $S$, we have that $\mathbb{E}_{p} f \geq \mathbb{E}_{p} g$ for all $p \in \pi(g)$.

If it is not the case that $f E g \sim g$ for some $E \in \Pi_{1}$, then either $f E g>g$ or $f E g \notin \mathcal{R}(g)$, both implying $\mathbb{E}_{p} f E g>\mathbb{E}_{p} g$ for all $p \in \pi(g)$. Hence, if it is not the case that $f E g \sim g$ for some $E \in \Pi_{1}$, we have that $\mathbb{E}_{p} f>\mathbb{E}_{p} g$ for all $p \in \pi(g)$. By the definition of $\pi^{w}(g)$, there exists $a \in(0,1)$ such that $a f+(1-a) g \succ g$, hence information is weakly valuable.

Suppose now that for all $E \in \Pi_{1}, f E g \sim g \succ f$. Note that $\sum_{E \in \Pi_{1}} \frac{1}{k} f E g=$ $\frac{1}{k} f+\frac{k-1}{k} g$, where $k$ is the number of $\Pi_{1}$ 's partition cells. Because $f E g \succsim g$ for each $E \in \Pi_{1}$, Axiom 4 implies $\frac{1}{k} f+\frac{k-1}{k} g \succsim g$. By setting $a=\frac{1}{k}$, information is weakly valuable.

Conversely, suppose that information is weakly valuable and Axiom 7 is satisfied. Using Corollary 1, we only need to show that Axiom 18 is satisfied. Suppose that for some event $E \in \mathcal{E}$ and acts $f \in \mathcal{F}, g \in \mathcal{F}_{+}$, we have $f \succsim_{E, f} g, f={ }_{E^{c}} g$, $g \succ f$ but $f \in \mathcal{R}(g)$. Suppose $f$ is not strictly positive, so that for some set $A \subseteq E$ (because $g$ is strictly positive and $f=E_{E^{c}} g$ ), we have $f(s)=0$ if and only if $s \in A$. If $\mathbb{E}_{p} g>\mathbb{E}_{p} f$ for some $p \in \pi(g)$, Axiom 2 implies that we can find strictly positive act $f^{\prime} \in \mathcal{F}_{+}$, by infinitesimally increasing the payoff for all states in $A$, such that $f^{\prime} \succsim_{E, f} g, f^{\prime}={ }_{E^{c}} g, g \succ f^{\prime}$ but $f^{\prime} \in \mathcal{R}(g)$. If $\mathbb{E}_{p} g \leq \mathbb{E}_{p} f$ for all $p \in \pi(g)$, $f \in \mathcal{R}(g)$ implies $\mathbb{E}_{p} g=\mathbb{E}_{p} f$ for some $p \in \pi(g)$. By taking a convex combination of $f$ and $g$, with large weight on $f$, Axioms 2, 4 and the definition of $\pi$ imply that we can find a strictly positive $f^{\prime}$ such that $f^{\prime} \succsim_{E, f} g, f^{\prime}={ }_{E^{c}} g, g \succ f^{\prime}$ but $f^{\prime} \in \mathcal{R}(g)$.

From Axiom 10 we have $f^{\prime} \succsim_{E, f^{\prime}} g$, so wlog we set $f=f^{\prime}$. Because $f \in \mathcal{R}(g)$, we have that $\mathbb{E}_{p} g \geq \mathbb{E}_{p} f$ for some $p \in \pi(g)$. Construct the same decision problems, $\mathcal{D}_{1}$ and $\mathcal{D}_{2}$, as in the third paragraph of the proof. Because information is weakly 
valuable and $\Pi_{1}$ is finer than $\Pi_{2}$, we have that $\mathcal{D}_{1}$ is weakly more valuable than $\mathcal{D}_{2}$. This implies that $a f+(1-a) g \succsim g$ for some $a \in(0,1]$.

From Axiom 4 we have that, for all $b \in[0,1], b(a f+(1-a) g)+(1-b) g=$ $a b f+(1-a b) g \succsim g$, hence, for all $c \in(0, a], c f+(1-c) g \succsim g$. If $c f+(1-c) g \succ g$ for some $c$, then $\mathbb{E}_{p}(c f+(1-c) g)>\mathbb{E}_{p} g$ for all $p \in \pi(g)$, contradicting $\mathbb{E}_{p} g \geq \mathbb{E}_{p} f$ for some $p \in \pi(g)$. We therefore have $c f+(1-c) g \sim g$ for all $c \in(0, a]$. Because $g \succ f$, Axiom 7 is contradicted.

Proof of Proposition 2 Suppose subjective beliefs are updated using Bayes' rule. Fix event $E \in \mathcal{E}$ and $f \in \mathcal{F}_{+}$. Suppose $f \succsim^{*} g$ and $f={ }_{E^{c}} g$. Using Corollary 1, we have $g \in \mathcal{R}(f)$. Then, $\mathbb{E}_{p} f \geq \mathbb{E}_{p} g$ for some $p \in \pi(f)$. Axiom 8 and Lemma 2 imply that $p(E)>0$. Because subjective beliefs are updated using Bayes' rule and $f=E^{c} g$, we have $\mathbb{E}_{p_{E}} f \geq \mathbb{E}_{p_{E}} g$ and $p_{E} \in \pi_{E, f}(f)$. Axioms 1 and 3 imply $f \succsim_{E, f} g$.

Conversely, suppose Axiom 13 and that there exist $f \in \mathcal{F}_{+}, p \in \pi(f)$ with $p(E)>$ 0 and $p_{E} \notin \pi_{E, f}(f)$. Then, there exists act $g$ such that $g \succ_{E, f} f$ and $\mathbb{E}_{p_{E}} g=\mathbb{E}_{p_{E}} f$. From Axiom 5 we have $g E f \succ_{E, f} f$. Because $\mathbb{E}_{p_{E}}(g E f)=\mathbb{E}_{p_{E}} f$ and $g E f={ }_{E^{c}} f$, we have that $\mathbb{E}_{p}(g E f)=\mathbb{E}_{p} f$, hence $g E f \in \mathcal{R}(f)$. From Corollary 1 Axiom 13 implies Axiom 17, hence $f \succsim_{E, f} g E f$, a contradiction.

The following is used in the proof of Lemmas 3 and 4.

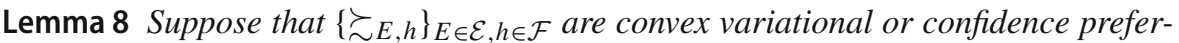
ences and satisfy Axiom 8, where $u$ is concave, increasing and differentiable. Suppose that for all $f$ and all $r \in M(f)$, if $r(E)>0$ then $r_{E} \in M_{E, f}(f)$. Then, Bayesian updating of subjective beliefs and Axiom 13 are satisfied.

Proof We show that there is Bayesian updating of subjective beliefs and then invoke Proposition 2, applied to strictly positive acts. Let $U: \mathbb{R}_{+}^{S} \rightarrow \mathbb{R}^{S}$ be the function $U(f)=(u(f(1), \ldots, u(f(S))))$, giving ex post utilities in each state. For any $f \in \mathbb{R}_{++}^{S}$, let $D U(f)$ be the $S \times S$ diagonal matrix with diagonal given by the vector of ex post marginal utilities $\left(u^{\prime}\left(f(1), \ldots, u^{\prime}(f(S))\right)\right)$. From Propositions 3 (for variational preferences) and 4 (for confidence preferences) in RSS, we have that $\pi(f)=\left\{\frac{q}{\|q\|}: q=p D U(f)\right.$ for some $\left.p \in M(f)\right\}$.

Suppose $q \in \pi(f)$ and $q(E)>0$ where, without loss of generality, $\|q\|=1$ and $q=r D U(f)$ for $r \in M(f)$. This means that $q(s)=r(s) u^{\prime}(f(s))$ for each $s \in S$. Because $r_{E} \in M_{E, f}(f)$,

we have $q_{E}^{\prime} \in \pi_{E, f}(f)$ where, for each $s \in E, q_{E}^{\prime}(s)=\frac{r(s) u^{\prime}(f(s))}{r(E)\left(\sum_{s^{\prime} \in E} \frac{r\left(s^{\prime}\right)}{r(E)} u^{\prime}\left(f\left(s^{\prime}\right)\right)\right)}=$ $\frac{r(s) u^{\prime}(f(s))}{\left(\sum_{s^{\prime} \in E} r\left(s^{\prime}\right) u^{\prime}\left(f\left(s^{\prime}\right)\right)\right)}=q_{E}(s)$, which implies $q_{E}^{\prime}=q_{E} \in \pi_{E, f}(f)$.

If $u$ is strictly increasing, then Axiom 8 is equivalent to requiring that the ambiguity index is finite only for beliefs that assign positive probability to all events in $\mathcal{E}$.

Lemma 9 If $u$ is strictly increasing, then Axiom 8 is equivalent to requiring that for all $E \in \mathcal{E}$ and all $p \in \Delta E, p(E)=0$ implies $c_{E, h}(p)=+\infty$. 
Proof of Lemma 9 Fix $F, E \in \mathcal{E}$ with $F \subseteq E$ and $h \in \mathcal{F}$. We then need to show that the following are equivalent.

- $c_{E, h}(p) \neq+\infty$ for some $p \in \Delta E$ such that $p(F)=0$,

- $F$ is weakly $\succsim_{E, h}$-null.

Let $P_{F} \subseteq \Delta E$ be such that $p \in P_{F}$ implies $c_{E, h}(p) \neq+\infty$ and $p(F)=0$. Suppose $P_{F} \neq \emptyset$. Let $f_{F, k}$ be the act such that $f_{F, k}(s)=k>0$ if $s \in F$ and $f_{F, k}(s)=0$ otherwise. For any act $g$ and $p \in P_{F}$, note that $\int u(g) d p+c(p)=\int u\left(g+f_{F, k}\right) d p+$ $c(p)$ for all $k>0$. This implies that for big enough $k^{*}, M_{E, h}\left(g+f_{F, k^{*}}\right) \subseteq P_{F}$. Moreover, for all $k>0, g+\left(f_{F, k^{*}}+f_{F, k}\right) \sim_{E, h} g+f_{F, k^{*}}$. Suppose there exists $g^{\prime}$ with $g+f_{F, k^{*}}=F^{c} g^{\prime}$, such that $g^{\prime} \succ_{E, h} g+f_{F, k^{*}}$. Set $k^{\prime}=\max _{s \in F} g^{\prime}(s)$. From Axiom 3, $g+\left(f_{F, k^{*}}+f_{F, k^{\prime}}\right) \succsim_{E, h} g^{\prime}$. But this implies $g+\left(f_{F, k^{*}}+f_{F, k^{\prime}}\right) \succ_{E, h} g+f_{F, k^{*}, \mathrm{a}}$ contradiction. Therefore, $F$ is weakly $\succsim_{E, h}$-null.

Conversely, suppose $F$ is weakly $\succsim_{E, h}$-null. Then, there exists $g$ such that, for all $g^{\prime}$ with $g={ }_{F^{c}} g^{\prime}$, we have $g \succsim_{E, h} g^{\prime}$. Suppose that for each $p \in \Delta E, p(F)=0$ implies $c_{E, h}(p)=+\infty$. Then, $p \in M_{E, h}(f)$ implies $p(F)>0$, for all $f \in \mathcal{F}$. Because $\int u(g) d p+c(p)<\int u\left(g+f_{F, k}\right) d p+c(p)$ for all $p$ with $p(F)>0$ and some $k>0$, we have that $g+f_{F, k} \succ_{E, h} g$, a contradiction.

Proof of Lemma 3 To show convexity of $c_{E, f}$, we need to establish that $c_{E, f}(a p+$ $\left.(1-a) p^{\prime}\right) \leq a c_{E, f}(p)+(1-a) c_{E, f} c\left(p^{\prime}\right)$ for all $p, p^{\prime} \in \Delta E$. Using (1), we need to show that $\int u(f) d n_{E, f}\left(a p+(1-a) p^{\prime}\right)+c\left(n_{E, f}\left(a p+(1-a) p^{\prime}\right)\right) \leq$ $a\left(\int u(f) d n_{E, f}(p)+c\left(n_{E, f}(p)\right)\right)+(1-a)\left(\int u(f) d n_{E, f}\left(p^{\prime}\right)+c\left(n_{E, f}\left(p^{\prime}\right)\right)\right)$.

For the first rule, if $c_{E, f}(p), c_{E, f}\left(p^{\prime}\right) \neq+\infty$, then there exist $q, q^{\prime} \in \Delta S$ such that $p \otimes^{E} q, p^{\prime} \otimes^{E} q^{\prime} \in D^{f}$ and $c\left(p \otimes \otimes^{E} q\right), c\left(p^{\prime} \otimes^{E} q^{\prime}\right) \neq+\infty$. From Lemma 1 in Araujo et al. (2019) there exists $b$ such that the Bayesian update of $b p \otimes^{E} q+(1-b) p^{\prime} \otimes^{E} q^{\prime}$ is $a p+(1-a) p^{\prime}$. From the convexity of $c, c\left(b p \otimes^{E} q+(1-b) p^{\prime} \otimes^{E} q^{\prime}\right) \neq+\infty$. From the convexity of $D^{f}, b p \otimes^{E} q+(1-b) p^{\prime} \otimes^{E} q^{\prime} \in D^{f}$ and from the definition of the updating rule, $a p+(1-a) p^{\prime} \in D_{E}^{f}$ and $c_{E, f}\left(a p+(1-a) p^{\prime}\right) \neq+\infty$. But this means that $n_{E, f}\left(a p+(1-a) p^{\prime}\right)=n_{E, f}(p)=n_{E, f}\left(p^{\prime}\right)$, so the inequality is true. If $c_{E, f}(p)=+\infty$ or $c_{E, f}\left(p^{\prime}\right)+\infty$, then the inequality is trivially satisfied. For the second rule, again from Lemma 1 in Araujo et al. (2019) and because $p \otimes^{E} r(E)=$ $p^{\prime} \otimes^{E} r(E)$, we have that $n_{E, f}\left(a p+(1-a) p^{\prime}\right)=\left(a p+(1-a) p^{\prime}\right) \otimes^{E} r=$ $a p \otimes^{E} r+(1-a) p^{\prime} \otimes^{E} r$, so the inequality is true.

To show lower semi continuity, note that for all $p$ with $c_{E, f}(p) \neq+\infty$, for the first updating rule the first two and the last term are constant, whereas the third term is a linear function of $p$. For the second rule, $c_{E, f}(p)$ is the sum of continuous functions of $p$.

To show Bayesian updating of subjective beliefs, suppose that $q \in M(f)$ and $q(E)>0$. For both updating rules, $n_{E, f}\left(q_{E}\right)=r$ for some $r \in M(f)$ with $r(E)>0$ and $r_{E}=q_{E}$. Let $p_{E} \in \Delta E$ and denote $n_{E, f}\left(p_{E}\right)$ by $p$. We then have $\int u(f) d r+$ $c(r) \leq \int u(f) d p+c(p)$. By substituting from equation (1), we have that $\int u(f) d r_{E}+$ $c_{E, f}\left(r_{E}\right)+k_{E, f} \leq \int u(f) d p_{E}+c_{E, f}\left(p_{E}\right)+k_{E, f}$, hence $\int u(f) d q_{E}+c_{E, f}\left(q_{E}\right) \leq$ $\int u(f) d p_{E}+c_{E, f}\left(p_{E}\right)$ and $q_{E} \in M_{E, f}(f)$. From Lemma 8, Bayesian updating of subjective beliefs and Axiom 13 are satisfied. 
To show that $D^{f}=D$ for all $f \in \mathcal{F}$ and $p \in D$ for all $p$ with $c(p) \neq+\infty$, implies Axiom 11, suppose $f \succsim g$ and $f=E^{c} g$. This implies that $\int u(f) d p+$ $c(p) \geq \int u(g) d q+c(q)$, where $p \in M(f)$ and $q \in M(g)$. Suppose that $g \succ_{E, f} f$. Substituting from equation (1), we have that $U_{E, f}(f)=\int u(f) d r+c(r)-k_{E, f}$ for some fixed $r \in M(f)$ and $U_{E, f}(g)=\int u(f) d r+c(r)-k_{E, f}+\min _{t \in D_{E}}\left\{\int u(g) d t-\right.$ $\left.\int u(f) d t\right\}$. Because $g \succ_{E, f} f$, we have that $\int u(g) d t>\int u(f) d t$, for all $t \in D_{E}$. Because $p(E)>0$ for all $p$ with $c(p) \neq+\infty$ and $q \in D$, we have $q_{E} \in D_{E}$ and $\int u(g) d q_{E}>\int u(f) d q_{E}$. From $f={ }_{E^{c}} g, \int u(g) d q_{E}>\int u(f) d q_{E}$ implies that $\int u(g) d q+c(q)>\int u(f) d q+c(q) \geq \int u(f) d p+c(p)$. Because $p \in M(f)$ and $q \in M(g)$ we have $g \succ f$, a contradiction.

To show that $D^{f}=M(f)$, for all $f \in \mathcal{F}$, implies Axiom 12, suppose that $f \succsim_{E, f} g$ and $f=E_{E^{c}} g$. From the calculations of the previous paragraph, we have that $U_{E, f}(f) \geq U_{E, f}(g)$ if and only if $\min \left\{\int u(g) d t-\int u(f) d t\right\} \leq 0$, which $t \in D_{E}^{f}$

implies that $\int u(g) d t_{E} \leq \int u(f) d t_{E}$ for some $t_{E} \in D_{E}^{f}$. Since $D^{f}=M(f)$, there exists $t \in M(f)$ whose Bayesian update is $t_{E}$. Because $f={ }_{E^{c}} g$, we have that $U(f)=\int u(f) d t+c(t) \geq \int u(g) d t+c(t) \geq \int u(g) d q+c(q)=U(g)$, where $q \in M(g)$. This implies that $f \succsim g$.

To show that these updating rules do not imply Axiom 11 and therefore fail DC, consider the example of Sect. 1.1 with $S=\left\{s_{1}, s_{2}, s_{3}\right\}$ and $E=\left\{s_{1}, s_{2}\right\}$. The agent has MEU ex ante preferences with $u(x)=x$ and $C$ being the convex hull of the following three measures: $p_{1}=(0.24,0.33,0.43), p_{2}=(0.34,0.2,0.46)$ and $p_{3}=(0.21,0.68,0.11)$. In this example each $M\left(f_{i}\right)$ is a singleton, so that $M\left(f_{1}\right)=M\left(f_{4}\right)=\left\{p_{3}\right\}$ and $M\left(f_{2}\right)=M\left(f_{3}\right)=\left\{p_{2}\right\}$. The subjective beliefs consist of the normalized vectors $p D U\left(f_{i}\right)$, where $p \in M\left(f_{i}\right)$ and $D U\left(f_{i}\right)$ is a diagonal matrix, with a diagonal consisting of the ex post marginal utilities, in this case 1's. We then have that $\pi\left(f_{1}\right)=\pi\left(f_{4}\right)=\left\{p_{3}\right\}$ and $\pi\left(f_{2}\right)=\pi\left(f_{3}\right)=\left\{p_{2}\right\}$. Because $u$ is linear, $f_{1} \succ f_{2}$ and $M\left(f_{1}\right)=p_{3}$, where $f_{1}=(1,0,0)$ and $f_{2}=(0,1,0)$.

For the first updating rule, let $D^{f_{1}}$ be the convex hull of $p_{1}$ and $p_{3}$, hence $M\left(f_{1}\right) \subseteq$ $D^{f_{1}}$ and $D_{E}^{f_{1}}$ is the convex hull of $p_{1 E}$ and $p_{3 E}$. We then have that $U_{E, f_{1}}\left(f_{1}\right)=$ $\min _{f_{1}}\left\{\int u\left(f_{1}\right) d p+c_{E, f_{1}}(p)\right\}=\int u(f) d p_{3}+c\left(p_{3}\right)-k_{E, f_{1}}$ and $U_{E, f_{1}}\left(f_{2}\right)=$ $p \in D_{E}^{f_{1}}$

$\min _{p \in D_{E}^{f_{1}}}\left\{\int u\left(f_{2}\right) d p+c_{E, f_{1}}(p)\right\}=\int u(f) d p_{3}+c\left(p_{3}\right)-k_{E, f_{1}}+\min _{p \in D_{E}^{f_{1}}}\left\{\int u\left(f_{2}\right) d p-\right.$ $\left.\int u\left(f_{1}\right) d p\right\}$. The last term simplifies to $\min _{p \in D_{E}^{f_{1}}}\left\{u(1) p\left(s_{2}\right)-u(1) p\left(s_{1}\right)\right\}$. Because $p\left(s_{2}\right)>p\left(s_{1}\right)$ for all $p \in D_{E}^{f_{1}}$, we have $f_{2} \succ_{E, f_{1}} f_{1}$.

To show that Axiom 12 is violated, it is enough to show that $f_{2} \succ_{E, f_{2}} f_{1}$. Let $D^{f_{2}}$ be the convex hull of $p_{2}$ and $p_{3}$. Noting that $M\left(f_{2}\right)=\left\{p_{2}\right\}$, we have $U_{E, f_{2}}\left(f_{2}\right)=\min _{p \in D_{E}^{f_{2}}}\left\{\int u\left(f_{2}\right) d p+c_{E, f_{2}}(p)\right\}=\int u(f) d p_{2}+c\left(p_{2}\right)-k_{E, f_{2}}$ and $U_{E, f_{2}}\left(f_{1}\right)=\min _{p \in D_{E}^{f_{2}}}\left\{\int u\left(f_{1}\right) d p+c_{E, f_{2}}(p)\right\}=\int u(f) d p_{2}+c\left(p_{2}\right)-k_{E, f_{2}}+$ 
$\min \left\{\int u\left(f_{1}\right) d p-\int u\left(f_{2}\right) d p\right\}$. The last term simplifies to $\min \left\{u(1) p\left(s_{1}\right)-\right.$ $p \in D_{E}^{f_{2}}$ $p \in D_{E}^{f_{2}}$

$\left.u(1) p\left(s_{2}\right)\right\}=\frac{u(1)}{p_{3}(E)}\left(p_{3}\left(s_{1}\right)-u(1) p_{3}\left(s_{2}\right)\right)<0$, hence $f_{2} \succ_{E, f_{2}} f_{1}$ but $f_{1} \succ f_{2}$.

For the second updating rule we set $n_{E, f_{1}}(p)=p \otimes^{E} p_{3}$. Let $C_{E}$ be the Bayesian updates of all elements of $C$, given $E$, consisting of the convex hull of $p_{1 E}, p_{2 E}$ and $p_{3 E}$. Note that if $p \notin C_{E}$, then $c_{E, f_{1}}(p)=+\infty$. Applying (1) and excluding constant $k_{E, f_{1}}$ we have $U_{E, f_{1}}\left(f_{1}\right)=\min _{p \in C_{E}}\left\{p\left(s_{1}\right)+p\left(s_{1}\right) p_{3}(E)-p\left(s_{1}\right)\right\}=p_{3}\left(s_{1}\right)=0.21$ and $U_{E, f_{1}}\left(f_{2}\right)=\min _{p \in C_{E}}\left\{p\left(s_{2}\right)+p\left(s_{1}\right) p_{3}(E)-p\left(s_{1}\right)\right\}=\min _{p \in C_{E}}\left\{1-p\left(s_{1}\right)\left(1+p_{3}\left(s_{3}\right)\right)\right\}=$ $1-p_{2 E}\left(s_{1}\right)\left(1+p_{3}\left(s_{3}\right)\right)=0.3011$. Hence, $f_{2} \succ_{E, f_{1}} f_{1}$.

Proof of Lemma 4 Note that $\phi_{E, h}(p)$ is either 0 or equal to $\frac{\phi(r)}{k_{E, h}} \frac{\mathbb{E}_{p} u(h)}{\mathbb{E}_{r} u(h)}$, where $\frac{\phi(r)}{k_{E, h} \mathbb{E}_{r} u(h)}$ is constant. Hence, quasiconcavity and upper semicontinuouty are satisfied. Proposition 4 in RSS shows that $\pi(h)=\left\{\frac{q}{\|q\|}: q=p D U(h)\right.$ for some $\left.p \in M(h)\right\}$.

To show Bayesian updating of subjective beliefs, suppose that $q \in M(h)$ and $q(E)>0$. By construction, $q_{E} \in D_{E}^{h}$. Moreover, for all $p \in D_{E}^{h}, \frac{\mathbb{E}_{p} u(h)}{\phi_{E, h}(p)}=k_{E, h} \frac{\mathbb{E}_{r} u(h)}{\phi(r)}$ for some fixed $r \in M(h)$ with $r(E)>0$, so it is independent of $p$. Hence, $q_{E} \in$ $M_{E, h}(h)=L_{a_{E, h}}=D_{E}^{h}$. From Lemma 8, Bayesian updating of subjective beliefs and Axiom 13 are satisfied.

Suppose now that $D^{f}=D=L_{a}$, for all $f \in \mathcal{F}$, and for all $p \in D$ we have $p(E)>0, f \succsim g$ and $f=E^{c} g$. This implies that $\frac{\mathbb{E}_{p} u(f)}{\phi(p)} \geq \frac{\mathbb{E}_{q} u(g)}{\phi(q)}$, where $p \in$ $M(f)$ and $q \in M(g)$. Suppose that $g \succ_{E, f} f$. Substituting from (2) we have that $U_{E, f}(f)=\frac{k_{E, f}}{\phi(r)} \mathbb{E}_{r} u(f)$ for some $r \in M(f)$ and $U_{E, f}(g)=\frac{k_{E, f}}{\phi(r)} \mathbb{E}_{r} u(f) \min _{t \in D_{E}} \frac{\mathbb{E}_{t} u(g)}{\mathbb{E}_{t} u(f)}$. Because $g \succ_{E, f} f$, we have that $\mathbb{E}_{t} u(g)>\mathbb{E}_{t} u(f)$, for all $t \in D_{E}$. In particular, $\mathbb{E}_{q E} u(g)>\mathbb{E}_{q E} u(f)$ and, since $f=E^{c} g$, we have $\frac{\mathbb{E}_{q} u(g)}{\phi(q)}>\frac{\mathbb{E}_{q} u(f)}{\phi(q)} \geq \frac{\mathbb{E}_{p} u(f)}{\phi(p)}$, a contradiction.

To show that $D^{f}=M(f)$, for all $f \in \mathcal{F}$, implies Axiom 12, suppose that $f \succsim_{E, f} g$ and $f=E_{E^{c}} g$. From the calculations of the previous paragraph, we have that $U_{E, f}(f) \geq U_{E, f}(g)$ if and only if $\min _{t \in D_{E}^{f}} \frac{\mathbb{E}_{t} u(g)}{\mathbb{E}_{t} u(f)} \leq 1$, which implies that $\mathbb{E}_{t_{E}} u(g) \leq \mathbb{E}_{t_{E}} u(f)$ for some $t_{E} \in D_{E}^{f}$. Since $D^{f}=M(f)$, there exists $t \in M(f)$ whose Bayesian update is $t_{E}$. Because $f=E^{c} g$, we have that $U(f)=\frac{1}{\phi(t)} \mathbb{E}_{t} u(f) \geq$ $\frac{1}{\phi(t)} \mathbb{E}_{t} u(g) \geq \frac{1}{\phi(q)} \mathbb{E}_{q} u(g)=U(g)$, where $q \in M(g)$. This implies that $f \succsim g$.

We now show that if $D^{h}$ is a strict subset of $L_{a}$, Axiom 11 and therefore DC may be violated. Consider the example of Sect. 1.1, with $S=\left\{s_{1}, s_{2}, s_{3}\right\}$ and $E=\left\{s_{1}, s_{2}\right\}$. The agent has MEU ex ante preferences with $u(x)=x$ and $L_{a}$ being the convex hull of the following three measures: $p_{1}=(0.24,0.33,0.43), p_{2}=(0.34,0.2,0.46)$ and $p_{3}=(0.21,0.68,0.11)$. Note that MEU is a special case of confidence preferences, where $\phi(p)=1$ if $p \in L_{a}$ and 0 otherwise, whereas $a=1 / 2$. As we showed there, $f_{1} \succ f_{2}$ and $M\left(f_{1}\right)=p_{3}$, where $f_{1}=(1,0,0)$ and $f_{2}=(0,1,0)$.

Let $D^{f_{1}} \subsetneq L_{a}$ be the convex hull of $p_{1}$ and $p_{3}$, hence $M\left(f_{1}\right) \subseteq D$ and $D_{E}^{f_{1}}$ is the convex hull of $p_{1 E}$ and $p_{3 E}$. We then have that that if $p \in D_{E}, \phi_{E, f_{1}}(p)=$ $\frac{\phi\left(p_{3}\right)}{k_{E, f_{1}}} \frac{\mathbb{E}_{p} u\left(f_{1}\right)}{\mathbb{E}_{p_{3}} u\left(f_{1}\right)}$, otherwise $\phi_{E, f_{1}}(p)=0$. By construction, $L_{a_{E, f_{1}}}=D_{E}$. 
We have $U_{E, f_{1}}\left(f_{1}\right)=\min _{p \in D_{E}} \frac{1}{\phi_{E, f_{1}}(p)} \mathbb{E}_{p} u\left(f_{1}\right)=\min _{p \in D_{E}} \frac{k_{E, f_{1}}}{\phi\left(p_{3}\right)} \mathbb{E}_{p_{3}} u\left(f_{1}\right)=\frac{k_{E, f_{1}}}{\phi\left(p_{3}\right)}$ $\mathbb{E}_{p_{3}} u\left(f_{1}\right)$ and $U_{E, f_{1}}\left(f_{2}\right)=\min _{p \in D_{E}} \frac{1}{\phi_{E, f_{1}}(p)} \mathbb{E}_{p} u\left(f_{2}\right)=\min _{p \in D_{E}} \frac{k_{E, f_{1}}}{\phi\left(p_{3}\right)} \mathbb{E}_{p_{3}} u\left(f_{1}\right) \frac{\mathbb{E}_{p} u\left(f_{2}\right)}{\mathbb{E}_{p} u\left(f_{1}\right)}=$ $\frac{k_{E, f_{1}}}{\phi\left(p_{3}\right)} \mathbb{E}_{p_{3}} u\left(f_{1}\right) \min _{p \in D_{E}} \frac{p\left(s_{2}\right)}{p\left(s_{1}\right)}$. Because $p\left(s_{2}\right)>p\left(s_{1}\right)$ for all $p \in D_{E}$, we have $f_{2} \succ_{E, f_{1}}$ $f_{1}$.

Proof of Lemma 5 Suppose Axiom 15, $f \succsim_{\mathcal{A} \mathcal{A}}^{*} g$ and $f=E_{E^{c}} g$. This implies that $f \succsim a f+(1-a) g$ for all $a \in[0,1]$. Let $B=\{h \in \mathcal{A}: h=a f+(1-a) g, a \in[0,1]\}$ be a convex budget set. We then have that $f$ is optimal in $B$. Applying Axiom 15, we have $f \succsim_{E, f} g$ and Axiom 16 is satisfied.

Conversely, suppose Axiom 16 and take $(\succsim, E, f, B) \in \mathcal{D}$ with $g \in B$ and $f=E^{c}$ $g$. Because $f, g \in B$ and $B$ is convex, $a f+(1-a) g \in B$ for all $a \in[0,1]$. The optimality of $f$ implies that $f \succsim a f+(1-a) g$. Hence, $f \succsim_{\mathcal{A} \mathcal{A}}^{*} g$ and Axiom 16 implies $f \succsim_{E, f} g$, so that Axiom 15 is satisfied.

Proof of Lemma 6 Let $U: \mathbb{R}_{+}^{S} \rightarrow \mathbb{R}^{S}$ be the function $U(f)=(u(f(1), \ldots$, $u(f(S)))$ ), giving ex post utilities in each state. For any $f \in \mathbb{R}_{+}^{S}$, let $D U(f)$ be the $S \times S$ diagonal matrix with diagonal given by the vector of ex post marginal utilities $\left(u^{\prime}\left(f(1), \ldots, u^{\prime}(f(S))\right)\right)$. Let $D U(f)(s)$ be the $s$-th element of the diagonal. From Proposition 5 in RSS, the set of subjective beliefs is a singleton, given by $\pi(f)=\frac{\mathbb{E}_{\mu}\left[\phi^{\prime}\left(\mathbb{E}_{p} u \circ f\right) p D U(f)\right]}{\left\|\mathbb{E}_{\mu}\left[\phi^{\prime}\left(\mathbb{E}_{p} u \circ f\right) p D U(f)\right]\right\|}$, where $\left\|\mathbb{E}_{\mu}\left[\phi^{\prime}\left(\mathbb{E}_{p} u \circ f\right) p D U(f)\right]\right\|=$ $\sum_{s^{\prime} \in S} \mathbb{E}_{\mu}\left[\phi^{\prime}\left(\mathbb{E}_{p} u \circ f\right) p\left(s^{\prime}\right)\right] D U(f)\left(s^{\prime}\right)$.

Given event $E$ and act $f$, the subjective belief becomes $\pi_{E}(f)=$ $\frac{\mathbb{E}_{\mu_{E, f}}\left[\phi^{\prime}\left(\mathbb{E}_{p_{E}} u \circ f\right) p_{E} D U(f)\right]}{\left\|\mathbb{E}_{\mu_{E, f}}\left[\phi^{\prime}\left(\mathbb{E}_{p_{E}} u \circ f\right) p_{E} D U(f)\right]\right\|}$.

Let $q=\pi(f)$, with $q(E)>0$ and $q^{\prime}=\pi_{E, f}(f)$. We need to show that for each $s \in E, \frac{q(s)}{q(E)}=q^{\prime}(s)$, or that

$$
\begin{gathered}
\frac{\mathbb{E}_{\mu_{E, f}}\left[\phi^{\prime}\left(\mathbb{E}_{p_{E}} u \circ f\right) p_{E}(s)\right] D U(f)(s)}{\sum_{s^{\prime} \in E} \mathbb{E}_{\mu_{E, f}}\left[\phi^{\prime}\left(\mathbb{E}_{p_{E}} u \circ f\right) p_{E}\left(s^{\prime}\right)\right] D U(f)\left(s^{\prime}\right)} \\
=\frac{\mathbb{E}_{\mu}\left[\phi^{\prime}\left(\mathbb{E}_{p} u \circ f\right) p(s)\right] D U(f)(s)}{\sum_{s^{\prime} \in E} \mathbb{E}_{\mu}\left[\phi^{\prime}\left(\mathbb{E}_{p} u \circ f\right) p\left(s^{\prime}\right)\right] D U(f)\left(s^{\prime}\right)} .
\end{gathered}
$$

Set $k(s)=\frac{\mathbb{E}_{\mu_{E, f}}\left[\phi^{\prime}\left(\mathbb{E}_{p_{E}} u \circ f\right) p_{E}(s)\right]}{\mathbb{E}_{\mu_{E, f}}\left[\phi^{\prime}\left(\mathbb{E}_{p_{E}} u \circ f\right)\right]}=\frac{\mathbb{E}_{\mu}\left[\phi^{\prime}\left(\mathbb{E}_{p} u \circ f\right) p(s)\right]}{\mathbb{E}_{\mu}\left[\phi^{\prime}\left(\mathbb{E}_{p} u \circ f\right)\right] p(E)}$ for all $s \in E$. By substituting the equalities $\mathbb{E}_{\mu_{E, f}}\left[\phi^{\prime}\left(\mathbb{E}_{p_{E}} u \circ f\right) p_{E}(s)\right]=k(s) \mathbb{E}_{\mu_{E, f}}\left[\phi^{\prime}\left(\mathbb{E}_{p_{E}} u \circ f\right)\right]$ and $\mathbb{E}_{\mu}\left[\phi^{\prime}\left(\mathbb{E}_{p} u \circ f\right) p(s)\right]=k(s) \mathbb{E}_{\mu}\left[\phi^{\prime}\left(\mathbb{E}_{p} u \circ f\right)\right] p(E)$ in (5), Bayesian updating of subjective beliefs is satisfied. 


\section{References}

Al-Najjar, N.I., Weinstein, J.: The ambiguity aversion literature: a critical assessment. Econ. Philos. 25(03), 249-284 (2009)

Amarante, M., Filiz, E.: Ambiguous events and maxmin expected utility. J. Econ. Theory 134(1), 1-33 (2007)

Anscombe, F.J., Aumann, R.: A definition of subjective probability. Ann. Math. Stat. 34, 199-205 (1963)

Araujo, A., Chateauneuf, A., José, H.F., Bruno, H.: Updating pricing rules. Econ. Theory 68(2), 335-361 (2019)

Asano, T., Kojima, H.: Consequentialism and dynamic consistency in updating ambiguous beliefs. Econ. Theory 68(1), 223-250 (2019)

Blackwell, D.: Comparison of experiments. In: Proceedings of the Second Berkeley Symposium on Mathematical Statistics and Probability, pp. 93-102. University of California Press (1951)

Cerreia-Vioglio, S., Maccheroni, F., Marinacci, M., Montrucchio, L.: Uncertainty averse preferences. J. Econ. Theory 146(4), 1275-1330 (2011)

Chateauneuf, A., Faro, J.H.: Ambiguity through confidence functions. J. Math. Econ. 45(9-10), 535-558 (2009)

Dempster, A.P: A generalization of Bayesian inference. J. R. Stat. Soc. Ser. B (Methodol.) (1968)

Dominiak, A., Lefort, J.-P.: Unambiguous events and dynamic Choquet preferences. Econ. Theory 46(3), 401-425 (2011)

Dominiak, A., Duersch, P., Lefort, J.-P.: A dynamic Ellsberg urn experiment. Games Econ. Behav. 75(2), 625-638 (2012)

Eichberger, J., Kelsey, D.: Uncertainty aversion and dynamic consistency. Int. Econ. Rev. 37(3), 625-640 (1996)

Eichberger, J., Grant, S., Kelsey, D.: CEU preferences and dynamic consistency. Math. Soc. Sci. 49, 143-151 (2005)

Eichberger, J., Grant, S., Kelsey, D.: Randomization and dynamic consistency. Econ. Theory 62(3), 547-566 (2016)

Ellsberg, D.: Risk, ambiguity, and the savage axioms. Q. J. Econ. 75, 643-669 (1961)

Epstein, L.G.: An axiomatic model of non-Bayesian updating. Rev. Econ. Stud. 73(2), 413-436 (2006)

Epstein, L.G., Le Breton, M.: Dynamically consistent beliefs must be Bayesian. J. Econ. Theory 61(1), 1-22 (1993)

Epstein, L.G., Marinacci, M.: Mutual absolute continuity of multiple priors. J. Econ. Theory 137(1), 716720 (2007)

Epstein, L.G., Schneider, M.: Recursive multiple-priors. J. Econ. Theory 113(1), 1-31 (2003)

Ergin, H., Gul, F.: A theory of subjective compound lotteries. J. Econ. Theory 144(3), 899-929 (2009)

Fagin, R., Halpern, J.Y.: A new approach to updating beliefs. In: Bonissone, P.P., Henrion, M., Kanal, L.N., Lemmer, J.F. (eds.) Uncertainty in Artifical Intelligence, vol. 6, pp. 347-374. North-Holland, Amsterdam (1991)

Faro, J.H., Lefort, J.-P.: Dynamic objective and subjective rationality. Theor. Econ. 14(1), 1-14 (2019)

Galanis, S.: The value of information under unawareness. J. Econ. Theory 157, 384-396 (2015)

Galanis, S.: The value of information in risk-sharing environments with unawareness. Games Econ. Behav. 97, 1-18 (2016)

Galanis, S.: Speculative trade and the value of public information. J. Public Econ. Theory 23(1), 53-68 (2021)

Galanis, S., Kotronis, S.: Updating awareness and information aggregation. B.E. J. Theor. Econ., (forthcoming)

Galanis, S., Ioannou, C., Kotronis, S.: Information aggregation under ambiguity: theory and experimental evidence. Discussion Paper 20/05, City, University of London, London, UK, (November 2019). https:// openaccess.city.ac.uk/id/eprint/23527/

Geanakoplos, J.: Game theory without partitions, and applications to speculation and consensus. Cowles Foundation Discussion Paper, No. 914 (1989)

Ghirardato, P.: Revisiting Savage in a conditional world. Econ. Theory 20(1), 83-92 (2002)

Ghirardato, P., Siniscalchi, M.: Risk sharing in the small and in the large. J. Econ. Theory 175, 730-765 (2018)

Ghirardato, P., Maccheroni, F., Marinacci, M.: Differentiating ambiguity and ambiguity attitude. J. Econ. Theory 118(2), 133-173 (2004) 
Ghirardato, P., Maccheroni, F., Marinacci, M.: Revealed ambiguity and its consequences: updating. In: Mohammed, A., John H .(eds.) Advances in Decision Making Under Risk and Uncertainty, volume 42 of Theory and Decision Library, pp. 3-18. Springer (2008)

Gilboa, I., Schmeidler, D.: Maxmin expected utility with non-unique prior. J. Math. Econ. 18, 141-153 (1989)

Gilboa, I., Schmeidler, D.: Updating ambiguous beliefs. J. Econ. Theory 59, 33-49 (1993)

Grant, S., Kajii, A., Polak, B.: Preference for information and dynamic consistency. Theory Decis. 48(3), 263-286 (2000)

Green, J.R., Stokey, N.L.: Two representations of information structures and their comparisons. Technical report, Technical report no. 271: Economics series: Institute for Mathematical Studies in the Social Sciences, Stanford University (1978)

Hanany, E., Klibanoff, P.: Updating preferences with multiple priors. Theor. Econ. 2, 261-298 (2007)

Hanany, E., Klibanoff, P.: Updating ambiguity averse preferences. B.E. J. Theor. Econ. 9(1), 1-53 (2009)

Hansen, L.P., Sargent, T.J.: Robust control and model uncertainty. Am. Econ. Rev. Papers Proc. 91(2), 60-66 (2001)

Hayashi, T., Miao, J.: Intertemporal substitution and recursive smooth ambiguity preferences. Theor. Econ. 6(3), 423-472 (2011)

Hirshleifer, J.: The private and social value of information and the reward to incentive activity. Am. Econ. Rev. 61, 561-574 (1971)

Jaffray, J.-Y.: Bayesian updating and belief functions. IEEE Trans. Syst. Man Cybern. 1144-1152 (1992)

Jaffray, J.-Y.: Dynamic decision making with belief functions. In: Ronald R.Y., Janusz K., Mario F. (eds.) Advances in the Dempster-Shafer Theory of Evidence, pp. 331-352. Wiley, New York (1994)

Karni, E., Safra, Z.: Dynamic consistency in English auctions and expected utility theory. Working paper 13-86, Foerder Institute for Economic Research, Tel-Aviv University, Ramat Aviv, Israel (1986)

Klibanoff, P., Marinacci, M., Mukerji, S.: A smooth model of decision making under ambiguity. Econometrica 73(6), 1849-1892 (2005)

Klibanoff, P., Marinacci, M., Mukerji, S.: Recursive smooth ambiguity preferences. J. Econ. Theory 144(3), 930-976 (2009)

Kreps, D.: A representation theorem for "preference for flexibility". Econometrica 47(3), 565-77 (1979)

Li, J.: Preferences for partial information and ambiguity. Theor. Econ. 13, 1059-1094 (2020)

Maccheroni, F., Marinacci, M., Rustichini, A.: Ambiguity aversion, robustness, and the variational representation of preferences. Econometrica 74(6), 1447-1498 (2006a)

Maccheroni, F., Marinacci, M., Rustichini, A.: Dynamic variational preferences. J. Econ. Theory 128(1), 4-44 (2006b)

Machina, M.J.: Dynamic consistency and non-expected utility models of choice under uncertainty. J. Econ. Lit. 27(4), 1622-68 (1989)

Madrian, B.C., Shea, D.F.: The power of suggestion: inertia in 401 (k) participation and savings behavior. Q. J. Econ. 116(4), 1149-1187 (2001)

Harry, M.: Markowitz. Portfolio selection. Journal of Finance 7, 77-91 (1952)

Masatlioglu, Y., Efe, A.O.: Rational choice with status quo bias. J. Econ. Theory 121(1), 1-29 (2005)

Milgrom, P., Stokey, N.: Information, trade, and common knowledge. J. Econ. Theory 26, 17-27 (1982)

Nau, R.F.: Uncertainty aversion with second-order utilities and probabilities. Manag. Sci. 52(1), 136-145 (2006)

Nehring, K.: Capacities and probabilistic beliefs: a precarious coexistence. Math. Soc. Sci. 38(2), 197-213 (1999)

Ortoleva, P.: Status quo bias, multiple priors and uncertainty aversion. Games Econ. Behav. 69(2), 411-424 (2010)

Pires, P.: A rule for updating ambiguous beliefs. Theory Decis. 53(2), 137-152 (2002)

Rigotti, L., Shannon, C., Strzalecki, T.: Subjective beliefs and ex ante trade. Econometrica 76(5), 1167-1190 (2008)

Sagi, J.S.: Anchored preference relations. J. Econ. Theory 130(1), 283-295 (2006)

Samuelson, W., Zeckhauser, R.: Status quo bias in decision making. J. Risk Uncertain. 1(1), 7-59 (1988)

Sarin, R., Wakker, P.P.: Dynamic choice and nonexpected utility. J. Risk Uncertain. 17, 87-119 (1998)

Savage, L.J.: The Foundations of Statistics. Wiley (1954)

Schlee, E.E.: The value of information in efficient risk-sharing arrangements. Am. Econ. Rev. 91(3), 509$524(2001)$ 
Schmeidler, D.: Subjective probability and expected utility without additivity. Econometrica 57(3), 571-87 (1989)

Shafer, G.: A Mathematical Theory of Evidence. Princeton University Press, Princeton (1976)

Siniscalchi, M.: Bayesian updating for general maxmin expected utility preferences. Technical report, Discussion Paper 1366, Center for Mathematical Studies in Economics and Management Science, Northwestern University (2001)

Siniscalchi, M.: Two out of three ain't bad: a comment on "the ambiguity aversion literature: a critical assessment." Econ Philos 25(03), 335-356 (2009)

Siniscalchi, M.: Dynamic choice under ambiguity. Theor. Econ. 6(3), (2011)

Snow, A.: Ambiguity and the value of information. J. Risk Uncertain. 40(2), 133-145 (2010)

Takashi, H.: Intertemporal substitution, risk aversion and ambiguity aversion. Econ. Theory 25, 933-956 (2005)

Tobin, J.: Liquidity preference as behavior toward risk. Rev. Econ. Stud. 25, 65-86 (1958)

Vergopoulos, V.: Dynamic consistency for non-expected utility preferences. Econ. Theory 48(2), 493-518 (2011)

Wakker, P.: Non-expected utility as aversion to information. J Behav. Decis. Making 1, 169-175 (1988)

Walley, P.: Statistical Reasoning with Imprecise Probabilities. Chapman and Hall, London (1991)

Wang, T.: Conditional preferences and updating. J. Econ. Theory 108, 286-321 (2003)

Wasserman, L., Kadane, J.B.: Bayes' theorem for Choquet capacities. Ann. Stat. 18, 1328-1339 (1990)

Yaari, M.E.: Some remarks on measures of risk aversion and on their uses. J. Econ. Theory 1, 315-329 (1969)

Publisher's Note Springer Nature remains neutral with regard to jurisdictional claims in published maps and institutional affiliations. 